\title{
A novel numerical framework for self-similarity in plasticity: Wedge indentation in single crystals
}

Juul, K. J.; Niordson, C. F. ; Nielsen, K. L.; Kysar, J. W.

Published in:

Journal of the Mechanics and Physics of Solids

Link to article, DOI:

10.1016/j.jmps.2017.09.011

Publication date:

2018

Document Version

Peer reviewed version

Link back to DTU Orbit

Citation (APA):

Juul, K. J., Niordson, C. F., Nielsen, K. L., \& Kysar, J. W. (2018). A novel numerical framework for self-similarity in plasticity: Wedge indentation in single crystals. Journal of the Mechanics and Physics of Solids, 112, 667-684. https://doi.org/10.1016/j.jmps.2017.09.011

\section{General rights}

Copyright and moral rights for the publications made accessible in the public portal are retained by the authors and/or other copyright owners and it is a condition of accessing publications that users recognise and abide by the legal requirements associated with these rights.

- Users may download and print one copy of any publication from the public portal for the purpose of private study or research.

- You may not further distribute the material or use it for any profit-making activity or commercial gain

- You may freely distribute the URL identifying the publication in the public portal 


\section{Accepted Manuscript}

A novel numerical framework for self-similarity in plasticity: Wedge indentation in single crystals

K.J. Juul, C.F. Niordson, K.L. Nielsen, J.W. Kysar

PII:

S0022-5096(17)30297-1

DOI:

10.1016/j.jmps.2017.09.011

Reference:

MPS 3189

To appear in:

Journal of the Mechanics and Physics of Solids

Received date: $\quad 28$ April 2017

Revised date: 27 August 2017

Accepted date: $\quad 22$ September 2017

Please cite this article as: K.J. Juul, C.F. Niordson, K.L. Nielsen, J.W. Kysar, A novel numerical framework for self-similarity in plasticity: Wedge indentation in single crystals, Journal of the Mechanics and Physics of Solids (2017), doi: 10.1016/j.jmps.2017.09.011

This is a PDF file of an unedited manuscript that has been accepted for publication. As a service to our customers we are providing this early version of the manuscript. The manuscript will undergo copyediting, typesetting, and review of the resulting proof before it is published in its final form. Please note that during the production process errors may be discovered which could affect the content, and all legal disclaimers that apply to the journal pertain. 


\title{
A novel numerical framework for self-similarity in plasticity: Wedge indentation in single crystals
}

\author{
K. J. Juul ${ }^{a, *}$ C. F. Niordson ${ }^{\mathrm{a}}$, K. L. Nielsen ${ }^{\mathrm{a}}$, J. W. Kysar ${ }^{\mathrm{b}}$ \\ ${ }^{a}$ Department of Mechanical Engineering, Solid Mechanics, Technical University of \\ Denmark, DK-2800 Kgs. Lyngby, Denmark \\ ${ }^{b}$ Department of Mechanical Engineering, Columbia University, New York, NY 10027, \\ United States
}

\begin{abstract}
A novel numerical framework for analyzing self-similar problems in plasticity is developed and demonstrated. Self-similar problems of this kind include processes such as stationary cracks, void growth, indentation etc. The proposed technique offers a simple and efficient method for handling this class of complex problems by avoiding issues related to traditional Lagrangian procedures. Moreover, the proposed technique allows for focusing the mesh in the region of interest. In the present paper, the technique is exploited to analyze the well-known wedge indentation problem of an elastic-viscoplastic single crystal. However, the framework may be readily adapted to any constitutive law of interest. The main focus herein is the development of the self-similar framework, while the indentation study serves primarily as verification of the technique by comparing to existing numerical and analytical studies. In this study, the three most common metal crystal structures will be investi-
\end{abstract}

\footnotetext{
${ }^{*}$ Corresponding author

Email address: krjoju@mek.dtu.dk (K. J. Juul)
} 
gated, namely the face-centered cubic (FCC), body-centered cubic (BCC), and hexagonal close packed (HCP) crystal structures, where the stress and slip rate fields around the moving contact point singularity are presented. Keywords: Self-similarity, Crystal plasticity, Wedge indentation, Asymptotic fields

\section{Introduction}

2

15 ${ }_{16}$ solids. However, as discussed by Saito and Kysar (2011) and in more detail 17 below, the proper analogy for a non-flat indenter is with a quasi-statically 
propagating crack. Drugan and Rice (1984) and Drugan (1986) explained that for elastic-plastic materials that satisfy the maximum plastic work inequality, the asymptotic fields for stationary and quasistatically propagating cracks are different in both isotropic and anisotropic materials. Furthermore the asymptotic fields may change as a consequence of large rotations and deformations. Hence, care must be taken with such methods, especially for anisotropic materials.

In the present work, a general computational framework specialized for geometrically self-similar problems in elastic-plastic solids is developed. The framework does not, as the previously mentioned self-similar methods, rely on reference solutions nor is it restricted to specific material laws. As an example, the framework is applied to wedge indentation of elastic-plastic single crystals.

For more than three decades, investigations have shown both analytically and numerically, that the material behaviour during indentation involves complex elastic-plastic deformation with finite strains and rotations. The early studies were closely related to crack growth which shares similarities to the indentation problem. For example, the boundary value problem of a stationary crack tip is analogous to that of a flat punch indentation. Likewise, the boundary value problem of a quasi-statically closing crack is analogous to that of a nearly-flat wedge indenter where the contact point singularity (e.g. ${ }_{99}$ the point where the indenter loses contact with the surface as it impinges into a material) moves quasistatically along the surface. 
${ }_{62}$ ically through elastic-plastic materials that obey the maximum plastic work

Analytical investigations of the asymptotic behaviour around a singular point in the crack tip and wedge indentation fields have been conducted by e.g. Drugan et al. (1982); Drugan and Rice (1984); Drugan (1986); Rice (1987); Drugan (2001); Saito and Kysar (2011) based on an extension of slip line theory that assumes a linear elastic, ideally plastic behavior (rather than the rigid, ideally plastic behavior typically associated with slip tine theory) and also can account for the elastic and plastic anisotropy of the crystal mechanical response. The governing partial differential equations are hyperbolic so the analytical solution is obtained via the method of characteristics. As a consequence, the deforming domain is divided into sectors within which deformation is either elastic or is ideally plastic on a well-defined set of slip systems. The sectors are separated by different types of discontinuities on sector boundaries, depending on the specific problem at hand.

For indentation (or eracks), the asymptotic solutions near the contact point (or crack tip) singularities consist of angular sectors centered at the singular point. The stress state in both plastically and elastically deforming regions can be readily calculated. Special attention must be paid to the boundaries between the angular sectors that consist of radial lines emanating from the singular point. If the singular point is stationary the solutions admit stress and velocity discontinuities across the radial sector boundaries. However, singular points, and hence sector boundaries, that move quasistatically through elastic-plastic materials that obey the maximin plastic work nequality have solutions that admit velocity discontinuities but not stress 

77 (2001a,b).

${ }_{85}$ the span where the contact point moves). Obviously, such numerical issues

discontinuities (Drugan and Rice, 1984; Drugan, 1986). Thus, the asymptotic fields associated with stationary and quasistatically moving singularities are quite different. Saito and Kysar (2011); Saito et al. (2012); Sarac and Kysar (2017) showed that asymptotic fields for flat punches and nearlyflat wedge indenters have significant differences, with related experimental analyses (Kysar et al., 2010; Sarac et al., 2016). These studies were heavily inspired by Rice (1987) and Kysar (2001a,b) where the differences with regard to cracks were reported, with related experimental analyses of stationary cracks (Bastawros and Kim, 2000; Crone and Shield, 2001) as well as quasistatically growing cracks (Kysar, 2000; Kysar and Briant, 2002). Rice et al. (1990) was among the first to confirm the distinct material behaviour in the vicinity of both a stationary and quasi-static crack tip through numerical analysis, with other studies by Mesarovic and Kysar (1996); Kysar

Recently, Saito et al. (2012) conducted numerical studies of the wedge indentation process confirming the analytical predictions by Saito and Kysar (2011). However, these investigations (Rice et al., 1990; Saito et al., 2012) are based on traditional incremental Lagrangian frameworks that suffer from numerical difficulties such as developing contact interfaces as well as problems with modelling a moving singularity due to the incremental procedure (nøt to mention the problem of maintaining sufficient mesh resolution over

\footnotetext{
86 are undesired and compromise accuracy of results. Thus, the main goal of
} 
the present study is to develop a general numerical framework specialized for self-similar problems in plasticity that avoids the numerical issues of the traditional procedures. In the following, self-similarity is referred to as a process where the fields, such as stress and strain fields, do not change for an observer continuously changing magnification of the view at a problem dependent rate. For example, considering wedge indentation, the fields beneath the indenter remain of identical shape, but change magnitude when the indenter impinges deeper into the material. To verify the numerical procedure, results of wedge indentation into the face-centered cubic (FCC) crystal structure will be compared to the analytical and numerical work of Saito and Kysar (2011) and Saito et al. (2012). Additionally, in order to demonstrate the capability of the developed framework, new results are presented for body-centered cubic (BCC) and hexagonal close-packed (HCP) crystal structures and compared to the analytical results in Saito and Kysar (2011).

The paper is divided into the following sections: The wedge indentation problem, analytical solutions, and material model are outlined in Section 2, self-similarity and the numerical framework are derived in Section 3, verification and results are presented in Section 4, and finally some concluding remarks are given in Section 5. Index notation, including Einstein's summation convention, is used throughout and the notation $\left({ }^{\circ}\right)$ signifies a time 107 derivative. 


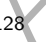

\section{Indentation with a nearly flat wedge indenter}

Quasi-static wedge indentation is chosen as the benchmark problem for the numerical framework developed as both analytical and numerical results exist for comparison (Saito and Kysar, 2011; Saito et al., 2012). Saito et al. (2012) considered indentation into a single metal crystal with a nearly flat wedge indenter such that, $\phi$, (cf. Fig. 1) approaches $0^{\circ}$. Here, friction between the indenter and the material is neglected and an elastic, ideally plastic single crystal with a very low critical resolved shear stress equal on all slip systems is assumed (see model parameters in Table 1). The proposed numerical framework is not limited to such extreme conditions, but this configuration ensures the conditions required for the analytical solutions developed by Saito and Kysar (2011). Additionally, this set-up allows for a two-dimensional (2D) plane strain analysis under a small strain assumption by employing effective in-plane slip systems that combine deformation on symmetric pairs of out-of-plane slip systems into an effective in-plane deformation. A detailed description and discussion of the effective slip systems can be found in Section 2.2.

A detailed study of the analytical solutions can be found in Saito and Kysar (2011) based on the extension of slip line theory that assumes linear elastic and ideally plastic behavior. Here, the FCC, BCC, and HCP crystal structures are treated for the 2D plane strain case. Saito and Kysar (2011) derived an analytical solution for a moving contact point singularity based on the assumption that stress discontinuities cannot exist in the deformation 
${ }_{152}$ ing radial rays. By adopting the slip systems in Table 2, it is seen that the 153

fields under these conditions (see Drugan and Rice, 1984). Following Rice (1987), the analytical investigation by Saito and Kysar (2011) showed that the asymptotic deformation fields consist of angular sectors centered at the singular point; the angular sectors can deform either elastically or plastically. The angular sectors are separated by radial rays emanating from the singular point that coincide either with the slip direction or the slip plane normal of the effective in-plane slip system. As described by Rice (1987), if the radial ray coincides with a slip direction, dislocations operate in glide shear along the ray and if the radial ray coincides with the slip plane normal dislocations operate in kink-shear mode. If the contact point singularity is stationary with respect to the crystal, the stress fields can admit stress jumps across the radial rays. However, if the contact point singularity moves quasistatically relative to the crystal, the angular sectors and sector boundaries move through the crystal as well. Under this condition, the stress fields do not admit discontinuities across the radial sector boundaries, but velocity discontinuities across the radial sector boundaries are allowed (Drugan and Rice, 1984).

The solution by Saito and Kysar (2011) for the asymptotic fields associated with the contact point singularity of a nearly-flat wedge impinging into an FCC crystal is reproduced in Fig. 2a. The solution consists of four elastically deforming angular sectors separated by three plastically deformglide shear is related to slip system (1) and (3), and the kink shear is related 
to slip system (2). The asymptotic solution for the BCC crystal is shown in Fig. 2b, having only three sectors separated by two plastically deforming rays (glide shear).

Saito and Kysar (2011) showed that the asymptotic solution for the stresses in the vicinity of the moving contact point singularity for the FCC and BCC crystals are described according to Eqs. (1)-(3) with $C_{1}=\sqrt{3} / 2$, $C_{2}=\sqrt{3}$, and $C_{3}=\sqrt{3} / 2$ for FCC, and $C_{1}=3 / 4, C_{2}=3 / 2$, and $C_{3}=3 / 4$ for BCC.

$$
\begin{aligned}
\frac{\sigma_{11}-\sigma_{22}}{2 \tau_{0}} & =C_{1} \sin (2 \theta) \\
\frac{\sigma_{11}+\sigma_{22}}{2 \tau_{0}} & =C_{2} \theta \\
\frac{\sigma_{12}}{\tau_{0}} & =C_{3}[1-\cos (2 \theta)]
\end{aligned}
$$

where $\sigma_{i j}$ is the stress tensor, $\tau_{0}$ is the critical resolved shear stress, and $\theta$ is the angle depicted in Fig. 2.

The analytical solutions of the stress field are presented in Figs. 7a and 9a for the FCC and BCC structures, respectively. The stress distribution is plotted as a function of the angle $\theta$ with $\theta=0$ at the undeformed surface in front of the moving contact point and $\theta=-\pi$ at the indenter surface going in a clockwise direction (see Fig. 2). Additionally, the analytical stress trajectory and yield surface are presented in Figs. $7 \mathrm{~b}$ and $9 \mathrm{~b}$ for the FCC and BCC structures, respectively. The yield surfaces are adopted directly from Table 2 through Table 4 in Saito and Kysar (2011). The last crystal 
structure of interest in this paper is the HCP structure. Saito and Kysar (2011) determined that the asymptotic solutions for the HCP crystal must include at least one plastic angular sector, unlike the FCC and BCC structures in which all angular sectors are elastic. Hence, an analytical solution of the stress field has not yet been derived for the HCP crystal (cf. Fig. 11b).

\subsection{Material model}

The plane strain study of indentation in single crystals is performed in a small strain setting. Thus, the total strain, $\varepsilon_{i j}$, is determined from the displacement, $u_{i}$, such that; $\varepsilon_{i j}=\left(u_{i, j}+u_{j, i}\right) / 2$ and furthermore the total strain is decomposed into the elastic part, $\varepsilon_{i j}^{e}$, and the plastic part, $\varepsilon_{i j}^{p}\left(\varepsilon_{i j}=\right.$ $\varepsilon_{i j}^{e}+\varepsilon_{i j}^{p}$ ). When the strain field (and its decomposition) are known, the stress field can be determined from the relationship; $\sigma_{i j}=\mathscr{L}_{i j k l}\left(\varepsilon_{k l}-\varepsilon_{k l}^{p}\right)$, where $\mathscr{L}_{i j k l}$ is the elastic stiffness tensor.

To determine the plastic part of the total strains for a single crystal, a summation over all slip systems, $\alpha$, is performed according to

$$
\dot{\varepsilon}_{i j}^{p}=\sum_{\alpha} \dot{\gamma}^{(\alpha)} P_{i j}^{(\alpha)}, \quad P_{i j}^{(\alpha)}=\frac{1}{2}\left(s_{i}^{(\alpha)} m_{j}^{(\alpha)}+m_{i}^{(\alpha)} s_{j}^{(\alpha)}\right)
$$

where $P_{i j}^{(\alpha)}$ is the Schmid tensor, $\dot{\gamma}^{(\alpha)}$ is the slip rate, and $s_{i}^{(\alpha)}$ and $m_{i}^{(\alpha)}$ are the unit vectors defining the slip direction and the slip plane normal, respectively (see Fig. 3). To determine the slip rate on each slip system, the following visco-plastic power law slip rate relation proposed by Hutchinson (1976) is 
adopted

$$
\dot{\gamma}^{(\alpha)}=\dot{\gamma}_{0} \operatorname{sgn}\left(\tau^{(\alpha)}\right)\left(\frac{\left|\tau^{(\alpha)}\right|}{g^{(\alpha)}}\right)^{1 / m}
$$

191 purposes of comparing the results of the computations to the existing analyti192 cal solution, but also because many detailed experiments are conducted under 193 nominally plane strain conditions in single crystals. However, the numerical

\subsection{Effective slip systems}

The reason for choosing a 2D plane stain model is mainly for verification where $\tau^{(\alpha)}=\sigma_{i j} m_{i}^{(\alpha)} s_{j}^{(\alpha)}$ is the resolved shear stress and $g^{(\alpha)}$ is the slip resistance. The slip resistance $g^{(\alpha)}=\tau_{0}^{(\alpha)}$ since only elastic, ideally plastic materials are considered in the present study.

The visco-plastic law in Eq. (5), implies that the rate-sensitivity of the material response increases for an increasing rate-sensitivity exponent, $m$, and vice versa, (N.B. The slip plane normal is denoted by unit vector $m_{i}$ whereas the rate-sensitivity exponent is denoted by the scalar $m$ ). Thus, for $m \rightarrow 0$, the constitutive material model approaches the rate-independent material response.

For the self-similar indentation problem dimensional analysis dictates that the indentation solution is governed by the following parameters

$$
\dot{\gamma}^{(\alpha)}\left(\frac{x_{i}}{a}\right)=F\left(\frac{\tau_{0}}{E}, \frac{\dot{a}}{a \dot{\gamma}_{0}}, \phi, m, \nu\right)
$$



${ }_{215}$ other three mirrored pairs. (3D) boundary value problems.

framework in Section 3 can equally well be exploited for three-dimensional

To ensure 2D plane strain deformation of single crystals, it is necessary to choose the plane of plane strain to coincide with a mirror symmetry plane in the crystal (see e.g. Rice, 1987; Kysar et al., 2005; Niordson and Kysar, 2014). Here, following Rice (1987), the (101) plane is chosen as the mirror symmetry plane for the plane strain deformation in the FCC and BCC crystals. The specimen geometry and the external loading must also have mirror symmetry about the crystallographic mirror plane. In that way the plastic slip systems can be grouped into mirrored pairs; both members of a pair share the same magnitude of resolved shear stress. Each of the two slip systems within a mirrored pair will then activate with the same slip rate, assuming the critical resolved shear stress is the same on both slip system. In this way the 12 slip systems from the FCC $\{111\}\langle 110\rangle$ family of slip systems reduces to 6 mirrored pairs of slip systems. For three of the mirrored pairs, the out-ofplane components of the plastic slip on one slip system will counteract that of the other slip system within the pair. The other three mirrored pairs do not have mutually canceling out-of-plane deformations, so the experiments and analyses are performed under conditions of small scale yielding (Rice, 1968) so that the elastically deforming region surrounding the plastic zone suppresses the out-of-plane deformation, and hence the activation, of these

Based on the crystal structure (see Fig. 3), three mirrored pairs of slip 

${ }_{238}$ strain deformation state. One of the mirrored pairs consists of $(\overline{1} 2 \overline{1})[111]$ 239

systems combine to form three effective plane strain slip systems in an FCC crystal, with each particular effective slip system denoted by $\alpha$ (the two underlying slip systems paired into the effective slip system are denoted $\alpha a$ and $\alpha b)$.

Referring to Fig. 3, effective slip system 1 has unit slip direction $s_{i}^{(1)}$ oriented at an angle of $\theta_{1}=\tan ^{-1}(\sqrt{2}) \approx 54.7356^{\circ}$ relative to the specimen $x_{1}$-axis. Effective slip system 2 has unit slip direction $s_{i}^{(2)}$ oriented at an angle of $\theta_{2}=0^{\circ}$ relative to the specimen $x_{1}$-axis. Effective slip system 3 has unit slip direction $s_{i}^{(3)}$ oriented at an angle of $\theta_{3} \neq \pi-\tan ^{-1}(\sqrt{2}) \approx 125.2644^{\circ}$ relative to the specimen $x_{1}$-axis. In the FCC crystal, effective slip systems 1 and 3 consist of a pair of coplanar slip systems whereas effective slip system 2 consists of a collinear pair of slip systems. In Kysar et al. (2005, 2010); Saito and Kysar (2011), the mirror plane was chosen equivalently to be (110), but the effective plane strain slip systems were oriented at the same respective angles for the orientation used herein.

Now considering a BCC crystal with crystallographic orientation of the specimen rotated by $90^{\circ}$ relative to that of the FCC crystal, as illustrated in Fig. 3. A BCC crystal has 24 different slip systems of type $\{1 \overline{1} 0\}\langle 111\rangle$ and $\{11 \overline{2}\}\langle 111\rangle$ (Hirth and Lothe, 1992). By choosing the (101) plane as the mirror symmetry plane for the plane strain deformation there are 12 mirrored pairs of slip system of which 6 pairs are capable of inducing a plane se strain deformation state. One of the mirrored pairs consts of (121)[111] and $(\overline{1} \overline{2} \overline{1})[1 \overline{1} 1]$. Since $s_{i}^{(\alpha)}$ and $m_{i}^{(\alpha)}$ for both slip systems lie within the $(\overline{1} 01)$ 
plane, both slip systems individually admit plane strain plastic deformation with $s_{i}^{(1)}$ oriented such that $\theta_{1}=\tan ^{-1}(\sqrt{2}) \approx 54.7356^{\circ}$ and $s_{i}^{(3)}$ oriented such that $\theta_{3}=\pi-\tan ^{-1}(\sqrt{2}) \approx 125.2644^{\circ}$ relative to the specimen $x_{1}$-axis. Another mirrored pair of slip systems consists of (101)[1̄11] and (101)[11̄1] which when activated in tandem produce an effective plain strain plastic slip system oriented such that $\theta_{2}=0^{\circ}$.

The remaining four mirrored pairs can be activated in tandem to form four effective in-plane slip systems, two of which have $s_{i}^{(\alpha)}$ parallel to $s_{i}^{(1)}$ and the other two of which have $s_{i}^{(\alpha)}$ parallel to $s_{i}^{(3)}$. Howeyer, the resolved shear stresses on these slip systems are smaller than that of effective slip systems 1 and 3 so these have been neglected in this analysis.

Lastly, for the HCP crystal, effective slips systems are not required when oriented such that the basal plane is the plane of deformation since three inplane slip systems exist for this configuration. The method for determining the effective slip systems are adopted from Rice (1987) and Niordson and Kysar (2014). The individual crystallographic slip systems and the corresponding effective slip systems are summarized in Table 2 and the orientation of the wedge indenter is shown in Fig. 3. The $s_{i}^{(\alpha)}$ and $m_{i}^{(\alpha)}$ of the individual crystallographic slip systems, in general, have components in the out-of-plane direction. In order to simplify the analytical and numerical analyses, (Rice, 1987) showed it convenient to treat each mirrored pairs of slip systems as an effective in-plane slip system with unit effective in-plane unit slip direction $S_{i}^{(\alpha)}$ and unit effective in-plane unit slip plane normal as $M_{i}^{(\alpha)}$. It is 
263

then necessary to scale the values of the critical resolved shear stress and the reference plastic strain rate for the effective slip systems.

To that end $\tau_{0}$ and $\dot{\gamma}_{0}$ are defined as the critical resolved shear stress and reference plastic strain rate, respectively, on the individual crystallographic plastic slip systems. Then $S_{i}^{(\alpha)}$ is substituted for $s_{i}^{(\alpha)}$ and $M_{i}^{(\alpha)}$ is substituted for $m_{i}^{(\alpha)}$ in Eq. (4) when calculating the resolved shear stresses. Finally, the effective critical resolved shear stresses and the effective reference plastic strain rates are scaled, respectively, with dimensionless scaling parameters $\lambda^{(\alpha)}$ and $\beta^{(\alpha)}$ to calculate the effective critical resolved shear stress, $\tau_{0}^{(\alpha)}$, and the effective reference strain rate, $\dot{\gamma}_{0}^{(\alpha)}$ for each of the effective in-plane slip systems according to

$$
\tau_{0}^{(\alpha)}=\lambda^{(\alpha)} \tau_{0}, \quad \text { and } \quad \dot{\gamma}_{0}^{(\alpha)}=\beta^{(\alpha)} \dot{\gamma}_{0}
$$

where the values of $\lambda^{(\alpha)}$ and $\beta(\alpha)$ for each effective slip system for FCC, BCC and HCP are listed in Table 2.

\section{Self-similarity and the numerical framework}

\subsection{Self-similar relation}

In the context of plasticity, self-similarity may be defined as solutions to a boundary value problem where field quantities remain unchanged in shape, and only the spatial extent of the solution scales with time or deformation. Such solutions may be encountered in indentation, void growth, and station- 
ary crack problems to name a few.

The governing equation to be derived in the following holds for any history dependent self-similar solution and as such can be exploited to address a wide range of problems. Here, considering indentation, self-similarity is obtained when the indentation rate, defined as $\dot{a} / a=\dot{c}$, is constant, where $a$ is the half contact length (i.e. the distance from the center of the indentation to the contact point singularity) and $\dot{a}$ the contact point velocity, as illustrated in Fig. 1.

Before the self-similar method is described further, two different coordinate systems applied in the derivation will be defined. The first is the reference coordinate system, $x_{i}$, that describes the position of all material points at time $t=0$ and the second is the self-similar coordinate system in which coordinates of material points change with time. The axes of the self-similar coordinate system expand and stretch accordingly with the evolution of the self-similar field. There exists a family of self-similar coordinate systems, all related by scaling factors, but a specific self-similar coordinate system where the coordinates are normalized with the half contact length, $a$, according to $\xi_{i}=x_{i} / a$ is employed here. Thus, the contact point singularity is located at $\xi_{i}=(1,0)$.

During indentation, self-similarity may be recognized by an observer who changes magnification in proportion to the indentation contact length, as this is the only characteristic length in the problem. Thus, any field quantity, $f$, must have the functional dependence $f\left(\xi_{i}\right)$. Under self-similar conditions, 
the only time dependence in the problem enters through the evolution of the characteristic length, $a$. Thus, the time rate of change of any field quantity in the self-similar coordinate system can be expressed through the following self-similar relation

$$
\dot{f}=\frac{\mathrm{d} f}{\mathrm{~d} t}=\frac{\partial f}{\partial \xi_{i}} \frac{\partial \xi_{i}}{\partial t}=-\frac{\dot{a}}{a} \xi_{i} \frac{\partial f}{\partial \xi_{i}}=-\dot{c} \xi_{i} \frac{\partial f}{\partial \xi_{i}}
$$

305

306 nate system, $x_{i}$, is given by $\left(\underline{g}_{1}, \underline{g}_{2}\right)$ such that the contact point at time $t=0$ 307 is located at $\left(a_{0}, 0\right)$. As indentation progresses, the basis in a self-similar 
coordinate system will be stretched according to $\left(\underline{G}_{1}^{(t)}, \underline{G}_{2}^{(t)}\right)=\left(\underline{g}_{1}, \underline{g}_{2}\right) a_{0} e^{i t}$, maintaining the contact point (singularity) at $\xi_{i}=(1,0)$. It then follows from the relation between bases (or equivalently the relation for the exponentially increasing contact length) that a material point and its history in the indentation process can be tracked according to $\xi_{i}=\left(x_{i} / a_{0}\right) e^{-\dot{c} t}$ in the self-similar coordinate system. The coordinates of a material point, $x_{i}$, in the self-similar coordinate system, $\xi_{i}$, therefore diminish with time.

With a suitable relation established between time derived and spatially derived quantities, a numerical integration technique similar to that of Dean and Hutchinson (1980) can be adopted. However, in contrast to the integration lines from Dean and Hutchinson (1980), which represent the material flow in a predefined direction, the integration technique here is based on spatial integration along lines starting far away in the elastic region, going towards the origin of the self-similar field (in this case the indenter tip), carrying the history dependence of material points (see illustration of a integration line in Fig. 1). As a consequence of the integration lines being located radially around the indenter tip, it is convenient to express the self-similar relation, Eq. (8), in a self-similar polar coordinate system with the origin located at the tip of the indenter. The self-similar expression can thus be expressed as $\dot{f}=-\dot{c} \rho \partial f / \partial \rho$ where $\rho$ is defined as the radial distance to a 328 point on the integration line. This self-similar relation will be employed in ${ }_{329}$ the development of the numerical framework. 
${ }^{347}$ where $T_{i}=\sigma_{i j} n_{j}$ is the surface traction with $n_{j}$ denoting the unit outward ${ }_{348}$ normal vector, $V$ is the volume, and $S$ is the bounding surface. Using the 349 finite element method, the PVW is discretized using a 2D 8-node isopara-

\subsection{Numerical framework}

The self-similar finite element model developed in the present study is a novel approach to handle this class of problems, inspired by the early work of Dean and Hutchinson (1980) for steady-state problems. The self-similar condition established in Section 3.1 states that any time derived quantity, $\dot{f}$, in the constitutive model can be directly related to a spatial derivative through the magnification rate, $\dot{c}$, according to the relation $\dot{f}=-\dot{c} \rho \partial f / \partial \rho$. Thus, any quantity of interest at a given material point, $\rho^{*}$, can be evaluated by integrating along a self-similar line, starting far away from the center of the self-similarity (in this case indenter tip) in the elastic zone, $\rho^{0}$, and ending at the point of interest closer to the indenter tip, $\rho^{*}$ (see integration path in Fig. 1). The point of interest, $\rho^{*}$, will then contain the load history of all points further away from the indenter tip. The self-similar integration procedure is performed with a classical forward Euler integration scheme.

As in Dean and Hutchinson (1980), the displacement field, $u_{i}$, is determined from the conventional principle of virtual work (PWV) for a quasistatic self-similar problem

$$
\int_{V} \mathscr{L}_{i j k l} \varepsilon_{k l} \delta \varepsilon_{i j} \mathrm{~d} V=\int_{S} T_{i} \delta u_{i} \mathrm{~d} S+\int_{V} \mathscr{L}_{i j k l} \varepsilon_{k l}^{p} \delta \varepsilon_{i j} \mathrm{~d} V
$$


metric elements with reduced Gauss integration $(2 \times 2$ Gauss points $)$.

The procedure for obtaining the self-similar solution is very similar to the one suggested by Juul et al. (2017) for a single crystal visco-plastic steadystate model, however, the integration is now carried out along lines emanating from the center of the self-similar field. The pseudo-algorithm for the selfsimilar procedure is as follows (superscript $n$ refers to the iterative step):

1. The plastic strains from the previous iteration, $\varepsilon_{i j}^{p(n-1)}$, are used to determine the current displacement field, $u_{i}^{(n)}$, from the PVW in Eq. (9) $\left(\varepsilon_{i j}^{p(n-1)}=0\right.$ in the first iteration).

2. The total strains, $\varepsilon_{i j}^{(n)}$, are determined from the displacement field, $u_{i}^{(n)}$.

3. The slip on each slip system and the plastic strain field are determined by the self-similar integration procedure.

(a) First the spatial derivatives of the slip and total plastic strains are determined by/applying the self-similar relation $(\partial f / \partial \rho=$ $-\dot{f} /(\rho \dot{c}))$

$$
\begin{aligned}
\frac{\partial \gamma^{(\alpha)}}{\partial \rho} & =-\frac{\dot{\gamma}_{0}^{(\alpha)}}{\rho \dot{c}} \operatorname{sgn}\left(\tau^{(\alpha)}\right)\left(\frac{\left|\tau^{(\alpha)}\right|}{g^{(\alpha)}}\right)^{1 / m} \\
\frac{\partial \varepsilon_{i j}^{p}}{\partial \rho} & =\sum_{\alpha} \frac{\partial \gamma^{(\alpha)}}{\partial \rho} P_{i j}^{(\alpha)}
\end{aligned}
$$

(b) Secondly, the current slip $\gamma^{(\alpha)(n)}$ on each slip system and the current plastic strains, $\varepsilon_{i j}^{p(n)}$, are determined by performing the selfsimilar integration 


$$
\gamma^{(\alpha)(n)}=\int_{\rho^{0}}^{\rho^{*}} \frac{\partial \gamma^{(\alpha)}}{\partial \rho} \mathrm{d} \rho, \quad \text { and } \quad \varepsilon_{i j}^{p(n)}=\int_{\rho^{0}}^{\rho^{*}} \frac{\partial \varepsilon_{i j}^{(p)}}{\partial \rho} \mathrm{d} \rho .
$$

4. The current stresses $\sigma_{i j}^{(n)}$ are then determined by applying the elastic constitutive relation; $\sigma_{i j}=\mathscr{L}_{i j k l}\left(\varepsilon_{k l}-\varepsilon_{k l}^{p}\right)$.

5. Step 1 through 4 is repeated, continuously feeding the new plastic field into the right hand side of Eq. (9), until convergence is obtained. Convergence is evaluated by comparing the displacement and stress field of the current iteration with the previous iteration.

It is noticed that the numerical framework is an iterative procedure (in contrast to the traditional incremental procedures), directly bringing out the self-similar state of the problem.

The stability of the self-similar framework is found to be very robust to various parameters, and eyen for very low rate-sensitivity exponents convergence will be obtained without any special approach to the problems. However, this version of the framework also relies on the modifications suggested by Niordson (2001) and Nielsen and Niordson (2012a), where substeps between the Gauss points are introduced in the spatial integration procedure which/increases the stability of the framework. 

${ }_{401}$ contour lines appear as rays emanating from the contact point. This is in 402

\section{Results}

The established numerical framework for self-similar problems is applied to the wedge indentation process to validate the solution for the stress and slip rate fields around the moving contact point (see also Saito et a1., 2012). The mesh is scaled around the contact point, such that the mesh is very fine in the vicinity of the contact point where a detailed view is desired, while the mesh is gradually coarser when moving away from the point of interest in order to save computational time (see illustration in Fig. 5).

\subsection{Stress Fields}

In this part, the stress distribution will be presented for the FCC, BCC, and HCP crystal structures as contour plots, angular variation around the moving contact point singularity, and as the stress trajectory in stress space. The contour plots are presented in the self-similar coordinate system, $\xi_{i}$, such that the contact point is always located at the coordinate $\xi_{i}=(1,0)$ (the contact point singularity).

Figure 6 presents contour plots of the stress components for the FCC crystal structure. According to Drugan and Rice (1984), stress discontinuities are not admissible across a quasi-statically moving surface in a plastically deforming material, which is also confirmed by the continuous stress distributions. Upon further inspection of the stresses, it is seen that some stress accordance with the asymptotic solutions in Eqs. (1)-(3). Moreover, it is 

23 a point corresponding to a radial sector boundary undergoing glide shear (i.e. ${ }_{424}$ a radial ray of plastically deforming material). Afterwards, the material again 425

noticed that the asymptotic solution breaks down some distance from the contact point as the field is no longer independent of the radius from the contact point.

To investigate the angular variation of the stresses around the contact point, the stresses have been extracted along an arc around the contact point using an inverse isoparametric mapping scheme. The extracted numerical values (markers) are plotted together with the analytical solution (lines) in Fig. 7. Here, the vertical lines represent the sector boundaries shown in Fig. 2a. It is seen that there is a good agreement between the analytical and numerical results both in terms of the angular development but also the magnitude. Furthermore, it is seen that the stress components satisfy the boundary conditions in terms of $\sigma_{12}=0$ and $\sigma_{22}=0$ at $\theta=0$ (the free undeformed surface) and $\sigma_{12}=0$ at $\theta=-180^{\circ}$ (the frictionless indenter surface).

Lastly, the stress trajectory is plotted in Fig. 7b, starting in the vicinity of $(0,0)$ which is at the free surface $(\theta=0)$ going in clockwise direction ending at the indenter surface. The elastic sectors (I-IV) are indicated on the stress trajectory according to Fig. 2a. The stress trajectory shows that the stresses start in an elastic region at the undeformed surface, then as $\theta \rightarrow-54.7^{\circ}$, the stresses develop into a state where the trajectory touches the yield surface at becomes elastic until a second radial sector boundary (this time undergoing 
${ }_{447}$ it is noticed that the numerical solution is, in fact, close to the yield surface, 448

kink shear) is encountered at $\theta=-90^{\circ}$ where the stresses just reach the yield surface and then become elastic again. At $\theta=-125.3^{\circ}$, the final radial sector boundary is encountered (glide shear) and the stress trajectory goes back to the initial point where the material behaviour is elastic. The fact that the material behaves elastically close to the indenter surface, indicates large stress triaxiality which is also confirmed by the stress component in Fig. 7a, where $\sigma_{11}=\sigma_{22}$ (Saito et al., 2012).

Figure 8 presents contour plots of the stresses for the BCC structure. Comparing to the solution for FCC (Fig. 6) similar features are observed for all stress components. Moreover, a similar asymptotic nature of the stresses only being dependent on the angle in the immediate vicinity of the moving contact point singularity is also valid. The stress components plotted along an arc around the contact point is shown in Fig. 9a for the BCC crystal, along with the analytical solutions from Eqs. (1)-(3). Again, the numerical solution is seen to be in good agreement with the analytical solution. In addition, the stress trajectory is presented in Fig. 9b for the BCC structure. Here, recall that it is expected to see one sector less than for the FCC structure as the analytical solution does not predict the existence of a radial sector boundary at $\theta=-90^{\circ}$ (Saito and Kysar, 2011). Starting in the vicinity of $(0,0)$ and moving in the clockwise direction, the first radial sector boundary (glide shear) is encountered. At the top horizontal line of the yield surface, even though this should not be the case for the BCC structure. This can 

${ }_{470}$ vertex to the right of the starting point. This indicates that the material ${ }_{471}$ behaves plastically within certain sectors as predicted by Saito and Kysar 
472 (2011).

\subsection{Slip rate fields}

The slip rate for the FCC, BCC, and HCP crystal structure will be presented in the following as contour plots near the moving contact point singularity (the same normalization of the axes as for the stresses is used). The main goal of this part is to bring forward the discontinuities expected in slip rate. These discontinuities were not directly seen in the previous results of the stress field since stress discontinuities are not admissible for a moving contact point singularity (Drugan and Rice, 1984). It should be noticed that the analytically proven discontinuities (Druganyand Rice, 1984; Rice, 1987; Saito and Kysar, 2011) will appear as rays with a finite width in the field of interest due to the rate dependent material model employed.

Figure 12 displays the normalized slip rate on the three effective slip systems for the FCC structure as well as the total slip rate (the sum $\dot{\gamma}^{(\text {tot })}=$ $\left.\sum^{\alpha}\left|\dot{\gamma}^{(\alpha)}\right|\right)$. According to Fig. 2a, a glide shear discontinuity should be observed at $\theta=-125.3^{\circ}$ on slip system (1) which is also the case (Fig. 12a). The numerical prédictions also holds for the two other analytical prediction by Saito and Kysar (2011) for slip system (2) which shows a kink shear ray at $\theta=-90^{\circ}$ (Fig. 12b) and lastly a glide shear ray on slip system (3) at $491 \mathrm{\theta}=-54.7^{\circ}$ (Fig. 12c). The glide shear ray in Fig. 12a is of particular in${ }_{492}$ terest as it is seen that the ray is reflected at the displacement symmetry ${ }_{493}$ boundary $\left(\xi_{1}=0\right)$, into a kink shear ray at $\theta=-125.3^{\circ}$. A better illus- 

${ }_{515}$ solution and its magnitude changes with the rate-sensitivity, $m$ (the same 516

tration of this is shown in Fig. 12d, where the sum of the slip rates on all systems is presented. It is seen that the kink shear ray arising from slip system (1) at point B is intersecting the kink shear ray on slip system (2) at point C. From a geometric point of view, these two rays should intersect on a line of $\theta=-64.7^{\circ}$, which is confirmed by the numerical results. Upon further inspection of the line OC, only slip system (1) contributes to plastic deformation below the line of $\theta=-64.7^{\circ}$, whereas all slip systems contribute to the deformation above the line. The observations for both the stress and the slip rate fields are consistent with the results obtained by Saito et al. (2012) for the FCC crystal.

In Fig. 13, the same results are presented for the BCC crystal structure. Since the BCC structure has slip systems identical to the FCC crystal, the same angles are observed (obviously, there is a change in the magnitudes, as the effective parameters are different). For slip system (1) (Fig. 13a), the glide shear ray is again observed at $\theta=-125.3^{\circ}$, and at $\theta=-54.7^{\circ}$ for slip system (3) (Fig. 13c). Moreover, the contour plot in Fig. 13b shows a kink shear ray at $\theta=-90^{\circ}$ similar to the one for the FCC structure, which according to the analytical solution should not exist. This is ascribed to the rate dependent model activating the kink shear due to the stress trajectory being very close to the yield surface (see Fig. 9b). Additionally, a small feature at approximately $\theta=-120^{\circ}$ in Fig. 13b is not part of the analytical feature is in fact seen for the FCC crystal in Fig. 12b, however, it is much 
smaller). For the BCC crystal a kink shear ray is also seen to emanate from the displacement symmetry boundary (Fig. 13d) perpendicular to the glide shear ray in slip system (1), making this system solely responsible for the plastic deformation below the OC line. Furthermore, it is also here observed that a kink shear ray, is reflected of the displacement symmetry boundary, and that it intersects the kink shear ray on slip system (2) on a $\theta=-64.7^{\circ}$ line in accordance with the geometrical expectation.

Lastly, the slip rate fields are presented for the HCP structure (Fig. 14) which deviate slightly from the FCC and BCC structures due to the difference in slip systems. Even though an analytical solution was not established for the HCP crystal, it is clear that the discontinuities coincide with the slip systems, where slip system (1) creates a glide shear ray at $\theta=-120^{\circ}$, a kink shear ray is formed on slip system (2) at $\theta=-90^{\circ}$, and finally another glide shear ray is formed at $\theta=-60^{\circ}$ on slip system (3). Besides the discontinuities related to the slip systems, an additional feature is observed in Fig. 14c at approximately $\theta=-80^{\circ}$. Similarly to the unexpected feature in Fig. 13b, this feature is expected to be an artifact of the rate dependency. As for the FCC and BCC crystal structures, a kink shear ray for slip system (1) reflects of the displacement symmetry boundary, however, it is much less pronounced for the HCP crystal. This kink shear ray should intersect the kink shear ray that emanates from slip system (2) at an angle of $\theta=-67^{\circ}$ (based on geometrical observations), however, the intersection is only vaguely observable from Fig. 14d. 


\section{Concluding remarks}

A novel numerical framework for self-similar problems in plasticity has been developed. The framework is specialized to this class of problems and eliminates a number of issues encountered when employing traditional Lagrangian procedures. Moreover, the framework readily enables focusing the mesh for high resolution of field solutions in the regions of interest.

Main focus in the presented work is on the development and verification of the self-similar framework. The verification is conducted by applying the newly developed framework to wedge indentation in a 2D small strain setting for single crystal plasticity, where both analytical (Saito and Kysar, 2011) and numerical (Saito et al., 2012) results exist for comparison. The framework, however, is general and holds for any self-similar problem in plasticity (also in $3 \mathrm{D}$ and with appropriate extensions for finite strains). The key findings are:

- The stress distribution in the vicinity of the contact point singularity corresponds to the analytical predictions by Saito and Kysar (2011) both qualitatively and quantitatively for the FCC and BCC crystal structure. Furthermore, the stress field for FCC is similar to the numerical results of Saito et al. (2012) showing the same qualitative features.

- Numerical simulation indeed reveals discontinuities in the slip rate corresponding to the predictions of Saito and Kysar (2011). Based on the 


\section{6. Acknowledgement}

${ }_{581}$ KJJ, CFN, and KLN are financially supported by The Danish Council for 582 Independent Research in the project "New Advances in Steady-State Engi-

analytical results both glide shear and kink shear sector boundaries exist for the FCC structure and this gives rise to three discontinuity lines emanating from the contact point singularity. The sector boundaries readily fall out by applying the new numerical framework.

- For the HCP crystal structure, an analytical expression was not constructed as the asymptotic solutions by Saito and Kysar (2011) were not admissible, implying that at least one plastic sector exists (only elastic sectors exist for FCC and BCC). The numerical results for the HCP crystal confirmed the existence of such sectors by having part of the stress trajectory remaining on the yield surface. Furthermore, both glide shear and kink shéar discontinuities are predicted by the numerical model for the HCP crystal.

For the BCC crystal, only glide shear discontinuities should exist according to analytical solutions, giving two sector boundaries. However, the numerical solutions also predict a third sector boundary corresponding to a kink shear discontinuity. The authors believe that this has to do with the rate dependent material model for which the kink shear discontinuity appears because the stress trajectory is very close to the yield surface. 
${ }_{583}$ neering Techniques", grant DFF-4184-00122. JWK gratefully acknowledges 584 support from NSF DMR-1310503. Prof. John W. Hutchinson is greatly 585 acknowledged for fruitful discussions concerning the self-similar framework. 586 Ph.D Chris Valentin Nielsen, DTU Mechanical Engineering, is acknowledged ${ }_{587}$ for his parallelization of the skyline solver module (Nielsen et al., 2012).

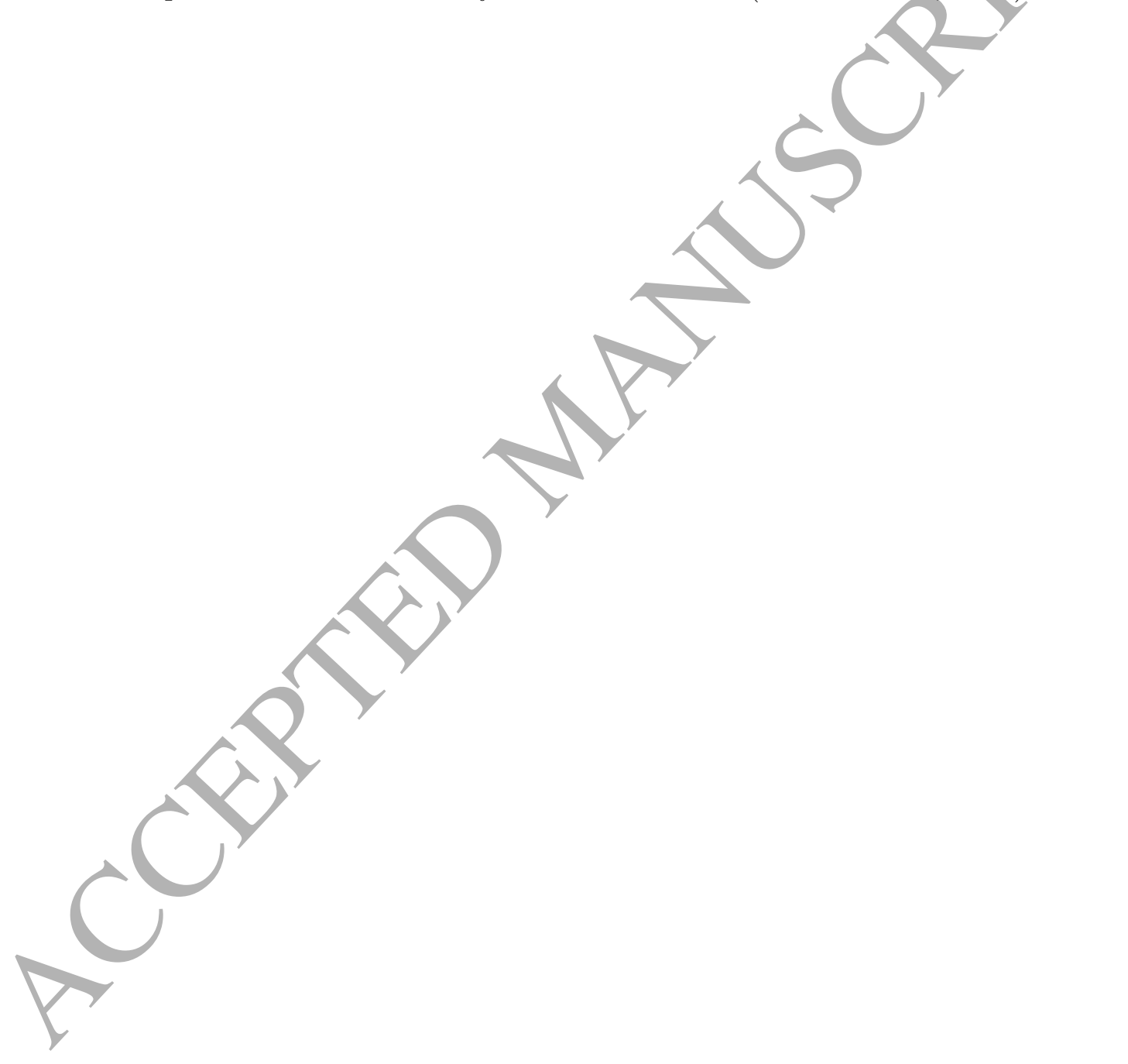




\section{References}

Bastawros, A. F., Kim, K.-S., 2000. Experimental analysis of near-crack-tip plastic flow and deformation characteristics (I): Polycrystalline aluminum. Journal of the Mechanics and Physics of Solids 48 (1), 67-98.

Biwa, S., Storåkers, B., 1995. An analysis of fully plastic brinell indentation. Journal of the Mechanics and Physics of Solids 43 (8), 1303-1333.

Bower, A. F., Fleck, N. A., Needleman, A., Ogbonna, N., 1993. Indentation of a power law creeping solid. Proceedings of the Royal Society of London Series A-mathematical Physical and Engineering Sciences 441 (1911), 97124.

Crone, W., Shield, T., 2001. Experimental study of the deformation near a notch tip in copper and copperberyllium single crystals. Journal of the Mechanics and Physics of Solids 49 (12), 2819 - 2838.

Dean, R. H., Hutchinson, J. W., 1980. Quasi-static steady crack growth in small-scale yielding. Fracture Mechanics: Twelfth Conference, ASTM STP700, American Society for Testing and Materials, 383-405.

Drugan, W. J., 1986. A more direct and general-analysis of moving strong discontinuity surfaces in quasi-statically deforming elasticplastic solids. J. Appl. Mech. 53, 224-226.

Drugan, W. J., 2001. Asymptotic solutions for tensile crack tip fields without 
kink-type shear bands in elastic-ideally plastic single crystals. J. Mech. Phys. Solids 49, 2155-2176.

Drugan, W. J., Rice, J. R., 1984. Restrictions on quasi-statically moving surfaces of strong discontinuity in elasticplastic solids. In: Dvorak, G.J., Shield, R.T. (Eds.), Mechanics of Material Behavior (The D.C. Drucker Anniversary Volume). Elsevier, 59-73.

Drugan, W. J., Rice, J. R., Sham, T. L., 1982. Asymptotic analysis of growing plane-strain tensile cracks in elastic ideally plastic solids. J. Mech. Phys. Solids 30, 447-473.

Hill, R., Storåkers, B., 1990. A concise treatment of axisymmetric indentation in elasticity. In: Eason, G., Odgen, R.W. (Eds.), Elasticity: Mathematical Methods and Applications (the 1.N. Sneddon 70th Birthday Volume). Ellis Horwood Limited, Chichester, England, 199-209.

Hirth, J. P., Lothe, J., 1992. Theory of Dislocations. Krieger Publishing Company, Malabar, Florida.

Hutchinson, J. W., 1976. Bounds and self-consistent estimates for creep of polycrystalline materials. Proc. R. Soc. Lond. A 348, 101-127.

Juul, K. J., Nielsen, K. L., Niordson, C. F., 2017. Steady-state crack growth in single crystals under mode i loading. J. Mech. Phys. Solids 101, 209-222. Kysar, J. W., 2000. Directional dependence of fracture in copper/sapphire bicrystal. Acta Materialia 48 (13), 3509-3524. 
Kysar, J. W., 2001a. Continuum simulations of directional dependence of crack growth along a copper/sapphire bicrystal interface. Part I: Experiments and crystal plasticity background. Journal of the Mechanics and Physics of Solids 49 (5), 1099-1128.

Kysar, J. W., 2001b. Continuum simulations of directional dependence of crack growth along a copper/sapphire bicrystal interface. Part/II: Crack tip stress/deformation analysis. Journal of the Mechanics and Physics of Solids 49 (5), 1129-1153.

Kysar, J. W., Briant, C. L., 2002. Crack tip deformation fields in ductile single crystals. Acta Materialia 50 (9), 2367-2380.

Kysar, J. W., Gan, Y. X., Mendez-Arzuza, G., 2005. Cylindrical void in a rigid-ideally plastic single crystal. Part I: Anisotropic slip line theory solution for face-centered cubic crystals. Int. J. Plas. 21, 1481-1520.

Kysar, J. W., Saito, Y., Öztop, M. S., Lee, D., Huh, W. T., 2010. Experimental lower bounds on geometrically necessary dislocation density. International Jøurnal of Plasticity 26 (8), 1097-1123.

Mesarovic, S. D., Kysar, J. W., 1996. Continuum aspects of directionally dependent cracking of an interface between copper and alumina crystals.

Mechanics of Materials 23 (4), 271-286.

${ }_{648}$ Nielsen, C. V., Zhang, W., Alves, L. M., Bay, N., Martins, P. A. F., 2012. Modeling of Thermo-Electro-Mechanical Manufacturing Processes with 
650

651 ${ }_{668}>1657$.

Applications in Metal Forming and Resistance Welding, First Edition. Springer.

Nielsen, K. L., Niordson, C. F., 2012a. Rate sensitivity of mixed mode interface toughness of dissimilar metallic materials: Studied at steady state. Int. J. Solids Struct. 49, 576-583.

Niordson, C. F., 2001. Analysis of steady-state ductile crack growth along a laser weld. Int. J. Frac. 111, 53-69.

Niordson, C. F., Kysar, J. W., 2014. Computational strain gradient crystal plasticity. J. Mech. Phys. Solids 62, 31-47.

Rice, J. R., 1968. Mathematical Analysis in the Mechanics of Fracture. In: Liebowitz, H. (Ed.), Fracture: An Advanced Treatise. Vol. Volume 2,. Academic Press, New York, Ch. 3, pp. 191-311.

Rice, J. R., 1987. Tensile crack tip fields in elastic-ideally plastic crystals. Mech. Mater. 6, 317-335.

Rice, J. R., Hawk, D. E., Asaro, R. J., 1990. Crack tip fields in ductile crystals. Int. J. Frac. 42, 301-322.

Saito, Y., Kysar, J. W., 2011. Wedge indentation in to elastic-plastic single crystals, 1: Asymptotic fields for nearly-flat wedge. Int. J. Plas. 27, 1640- 
669

Saito, Y., Oztop, M. S., Kysar, J. W., 2012. Wedge indentation in to elasticplastic single crystals, 2: Simulations for face-centered cubic crystals. Int. J. Plas. 28, 70-87.

Sarac, A., Kysar, J. W., 2017. Experimental validation of plastic constitutive hardening relationship based upon the direction of the net burgers density vector. Journal of the Mechanics and Physics of Solids Under Review.

Sarac, A., Oztop, M. S., Dahlberg, C. F. O., Kysar, J. W., 2016. Spatial distribution of the net Burgers vector density in a deformed single crystal. International Journal of Plasticity 85, 110 - 129.

Storåkers, B., Larsson, P. L., 1994. On brinell and boussinesq indentation of creeping solids. Journal of the Mechanics and Physics of Solids 42 (2), $307-332$.

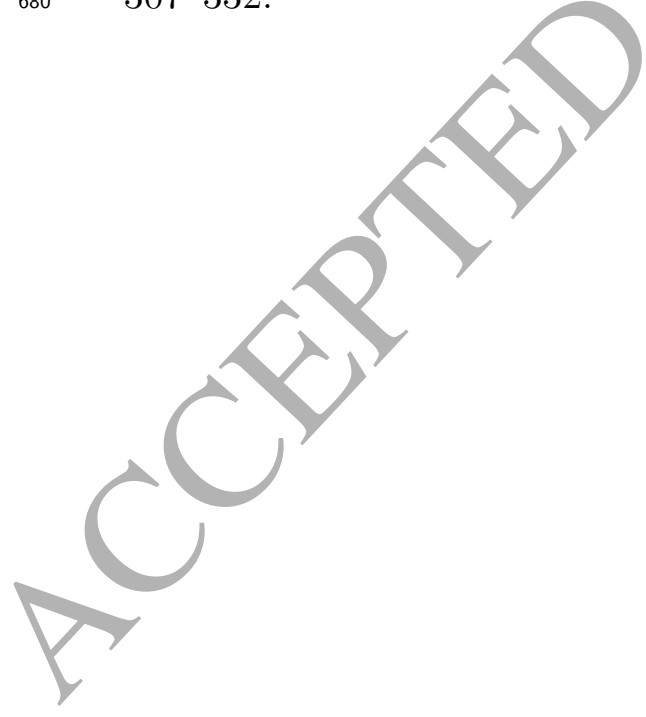




\section{List of Figures}

1 Wedge indentation in a rate dependent single crystal. Inside the self-similar history dependent domain (SS domain) the developed numerical framework is applied, whereas outside this domain, the material is treated as being linear elastic.

2 Sector structure for asymptotic fields beneath the contact point singularity (a) with kink shear sector boundary (FCC) and (b) without kink shear sector boundary (BCC) (Saito and Kysar, 2011). The parameter $\alpha=54.7^{\circ}$ for both the FCC and BCC crystal structures. . . . . . . . . . . . . . . . 38

3 Crystallographic orientation of the specimen relative to the wedge indenter, and the effective slip systems for the FCC, BCC, and HCP crystal structures, respectively.

4 Development of self-similarity through time in (a) the reference coordinates with the basis vectors $\left(g_{4}, g_{2}\right)$, and in (b) the self-similar coordinate system with the stretched basis vectors $\left(G_{1}^{(t)}, G_{2}^{(t)} \ldots \ldots \ldots \ldots \ldots \ldots\right.$

5 Right hand side of the domain used for numerical simulations. The dashed arrows indicate the direction of gradually increasing element size. The boundary of the domain is sufficiently far away from the contact point to have negligible influence on the results (the boundary is clamped). . . . . . . . . . 41

6 Stress distribution around the right hand side contact point, $\xi_{i}=(1,0)$, in FCC crystal for the stress components (a) $\sigma_{11} / \tau_{0}$, (b) $\sigma_{22} / \tau_{0}$ and $\left(\right.$ c) $\sigma_{12} / \tau_{0} \ldots \ldots \ldots \ldots$. . . . . . . . . 42

7 Stress distribution for FCC around the right hand side contact point projected as (a) angular distribution, and (b) stress trajectory with the thick line representing the yield surface. The lines represent the analytical solution while the markers indicate the numerical results. . . . . . . . . . . . . 43

8 Stress distribution around the right hand side contact point, $\xi_{i}=(1,0)$, in BCC crystal for the stress components (a) $\sigma_{11} / \tau_{0}$, (b) $\sigma_{22} / \tau_{0}$ and (c) $\sigma_{12} / \tau_{0} \ldots \ldots \ldots . \ldots 44$ 
9 Stress distribution for BCC around the right hand side contact point projected as (a) angular distribution, and (b) stress trajectory with the thick line representing the yield surface. The lines represent the analytical solution while the markers indicate the numerical results. . . . . . . . . . . . . . 45

10 Stress distribution around the right hand side contact point, $\xi_{i}=(1,0)$, in HCP crystal for the stress components (a) $\sigma_{11} / \tau_{0},(\mathrm{~b}) \sigma_{22} / \tau_{0}$ and (c) $\sigma_{12} / \tau_{0}$.

11 Stress distribution for HCP around the right hand side contact point projected as (a) angular distribution, and (b) stress trajectory with the thick line representing the yield surface. Here, only the numerical solution is presented. . . . . . . . 47

12 Slip rate around the right hand side contact point, $\xi_{i}=(1,0)$, in FCC crystal for the slip systems (a) $\left|\dot{\gamma}^{(1)}\right| / \dot{c}$, (b) $\left|\dot{\gamma}^{(2)}\right| / \dot{c}$, (c) $\left|\dot{\gamma}^{(3)}\right| / \dot{c}$, and $(\mathrm{d}) \dot{\gamma}^{\text {(tot })} / \dot{c}\left(\dot{\gamma}^{\text {(tot })}=\sum^{\alpha}\left|\dot{\gamma}^{(\alpha)}\right|\right) \ldots \ldots . . . . .48$

13 Slip rate around the right hand side contact point, $\xi_{i}=(1,0)$, in BCC crystal for the slip systems (a) $\left|\dot{\gamma}^{(1)}\right| / \dot{c}$, (b) $\left|\dot{\gamma}^{(2)}\right| / \dot{c}$, (c) $\left|\dot{\gamma}^{(3)}\right| / \dot{c}$, and (d) $\dot{\gamma}^{\text {(tot) })} / \dot{c}\left(\dot{\gamma}^{\text {tot })}=\sum^{\alpha}\left|\dot{\gamma}^{(\alpha)}\right|\right) . \ldots \ldots . . . . \quad 49$

14 Slip rate around the right hand side contact point, $\xi_{i}=(1,0)$, in HCP crystal for the slip systems (a) $\left|\dot{\gamma}^{(1)}\right| / \dot{c}$, (b) $\left|\dot{\gamma}^{(2)}\right| / \dot{c}$, (c) $\left|\dot{\gamma}^{(3)}\right| / \dot{c}$, and (d) $\dot{\gamma}^{\text {(tot })} / \dot{\boldsymbol{c}}\left(\dot{\gamma}^{(\text {tot })}=\sum^{\alpha}\left|\dot{\gamma}^{(\alpha)}\right|\right) . \ldots . . .50$ 


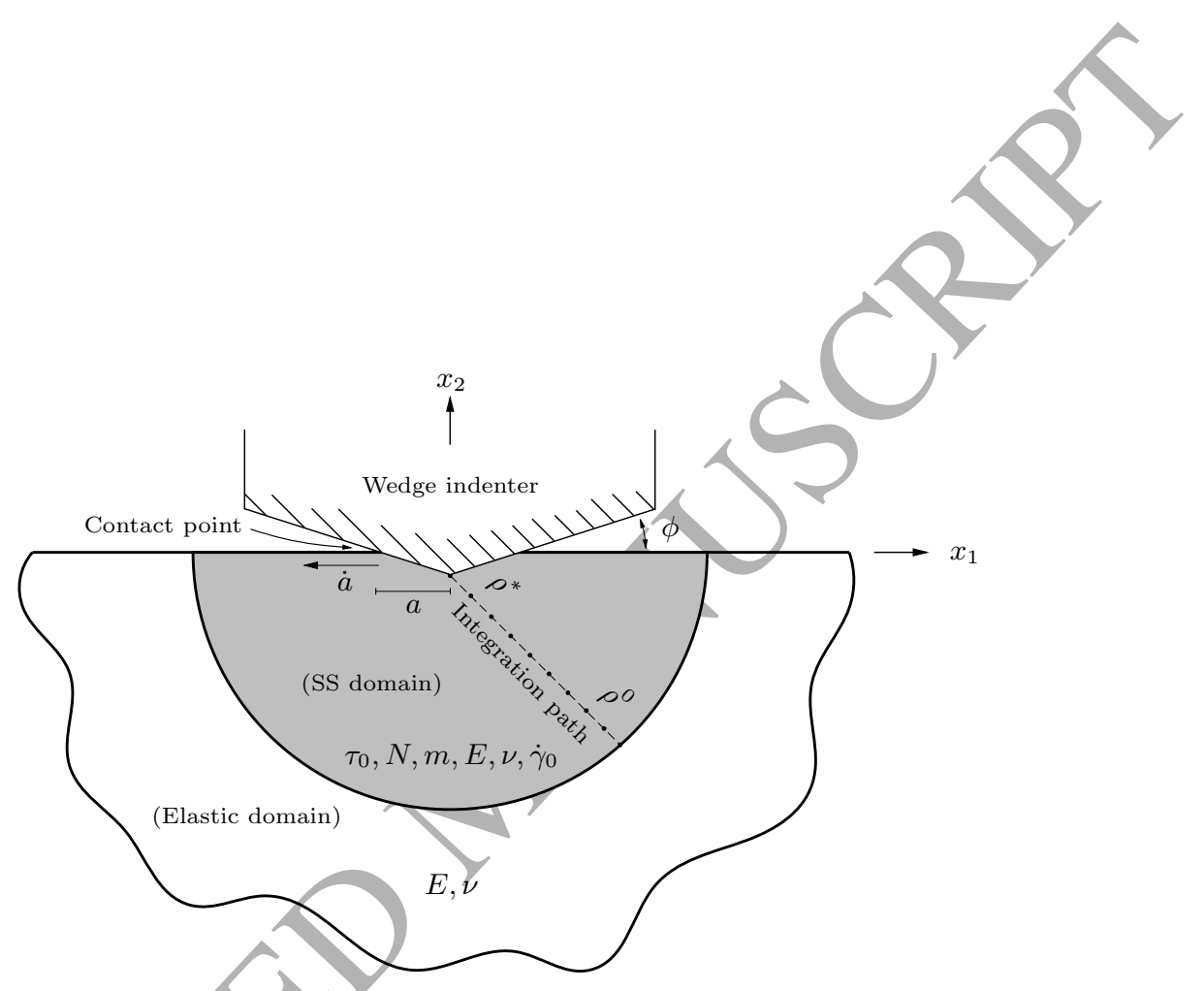

Figure 1: Wedge indentation in a rate dependent single crystal. Inside the selfsimilar history dependent domain (SS domain) the developed numerical framework is applied, whereas outside this domain, the material is treated as being linear elastic.

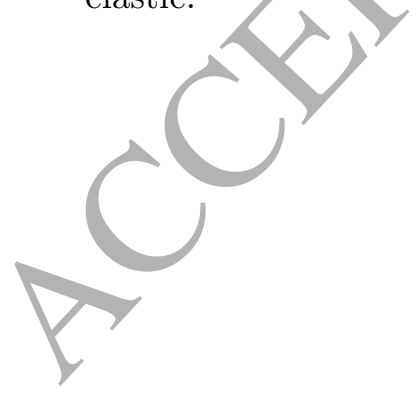




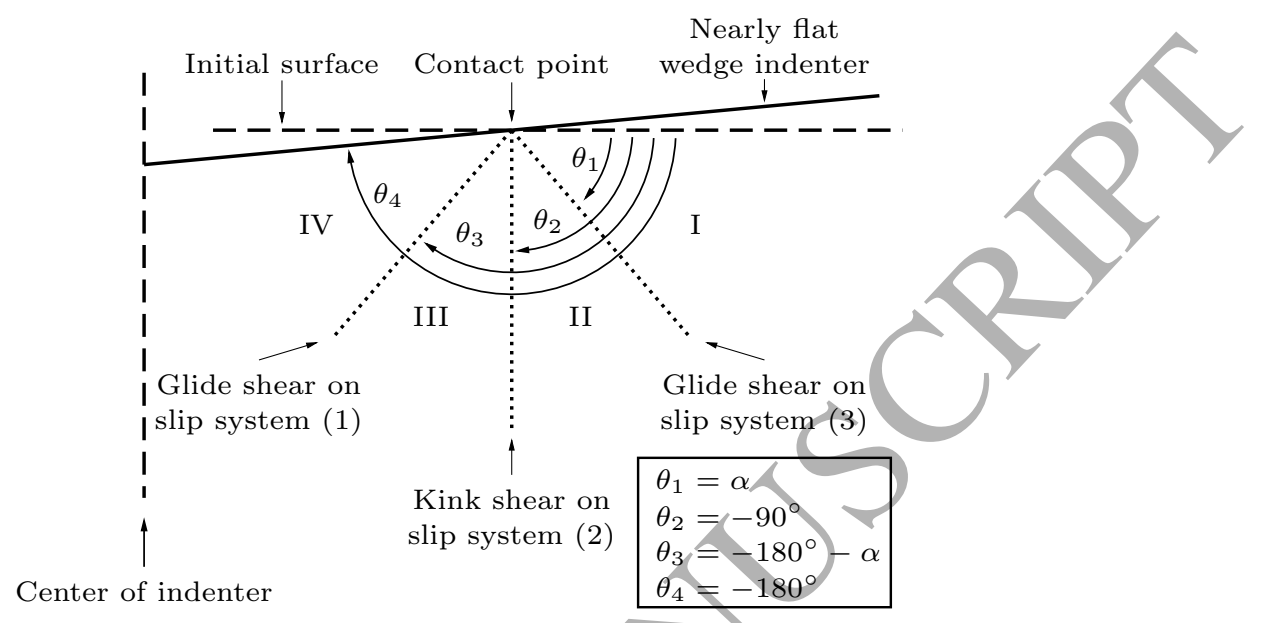

(a)

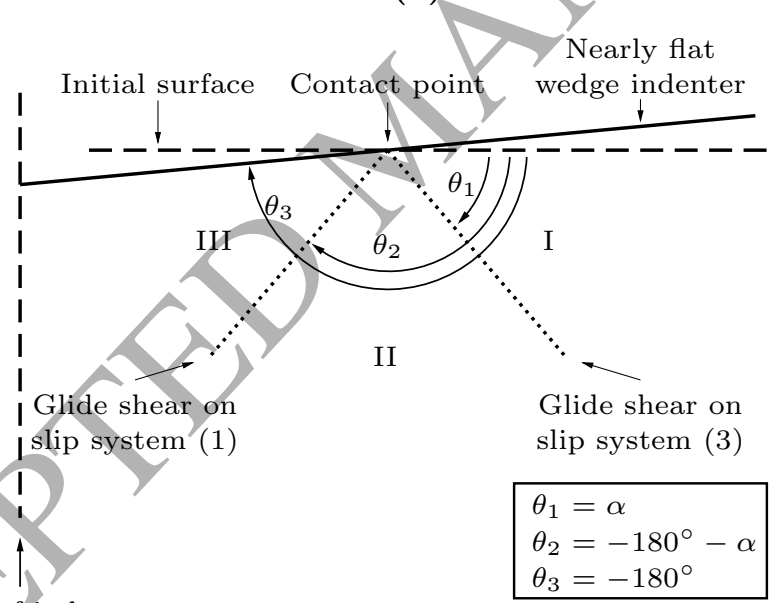

Center of indenter

(b)

Figure 2: Sector structure for asymptotic fields beneath the contact point singularity (a) with kink shear sector boundary (FCC) and (b) without kink shear sector boundary (BCC) (Saito and Kysar, 2011). The parameter $\alpha=54.7^{\circ}$ for both the FCC and BCC crystal structures. 

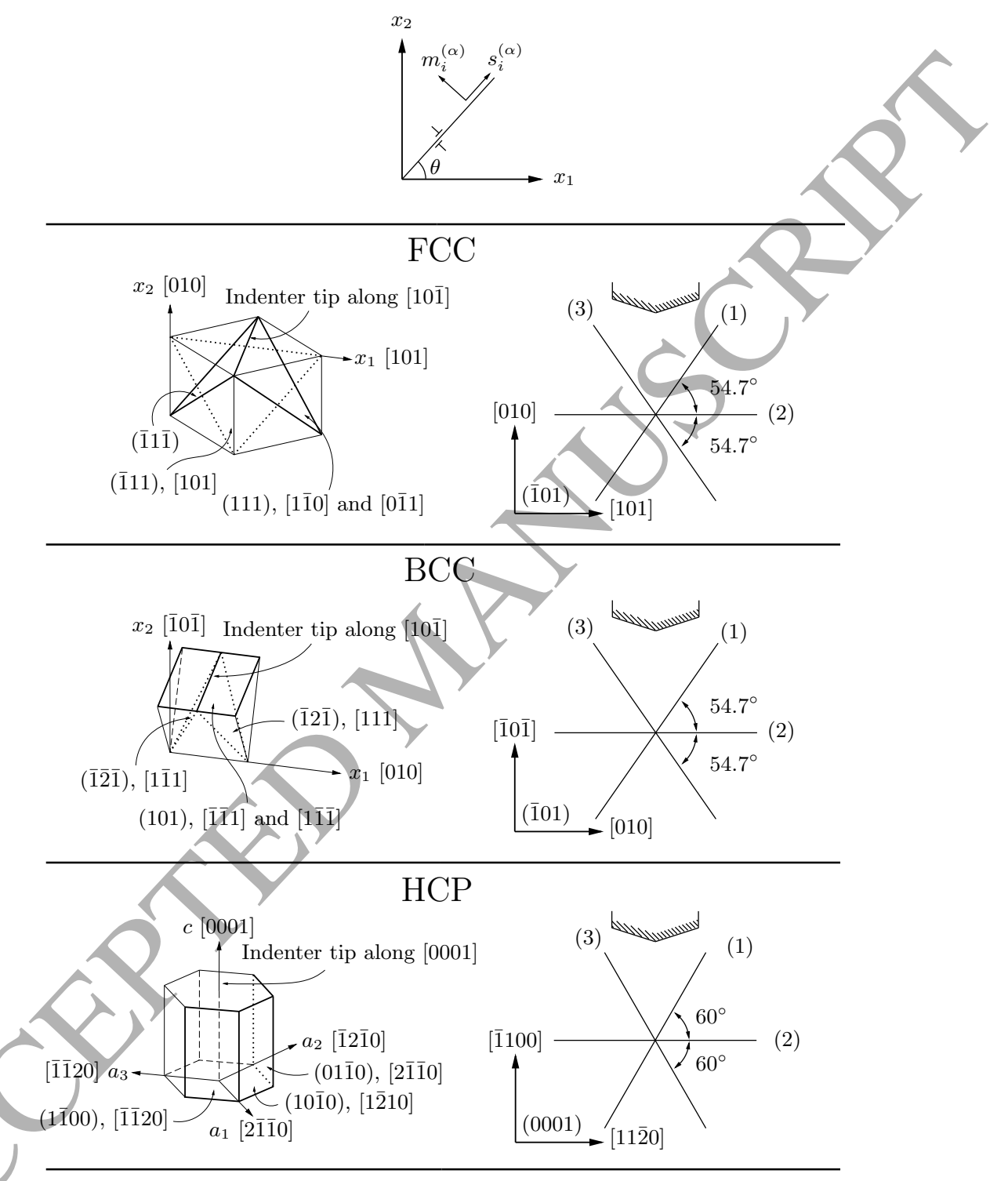

Figure 3: Crystallographic orientation of the specimen relative to the wedge indenter, and the effective slip systems for the FCC, BCC, and HCP crystal structures, respectively. 

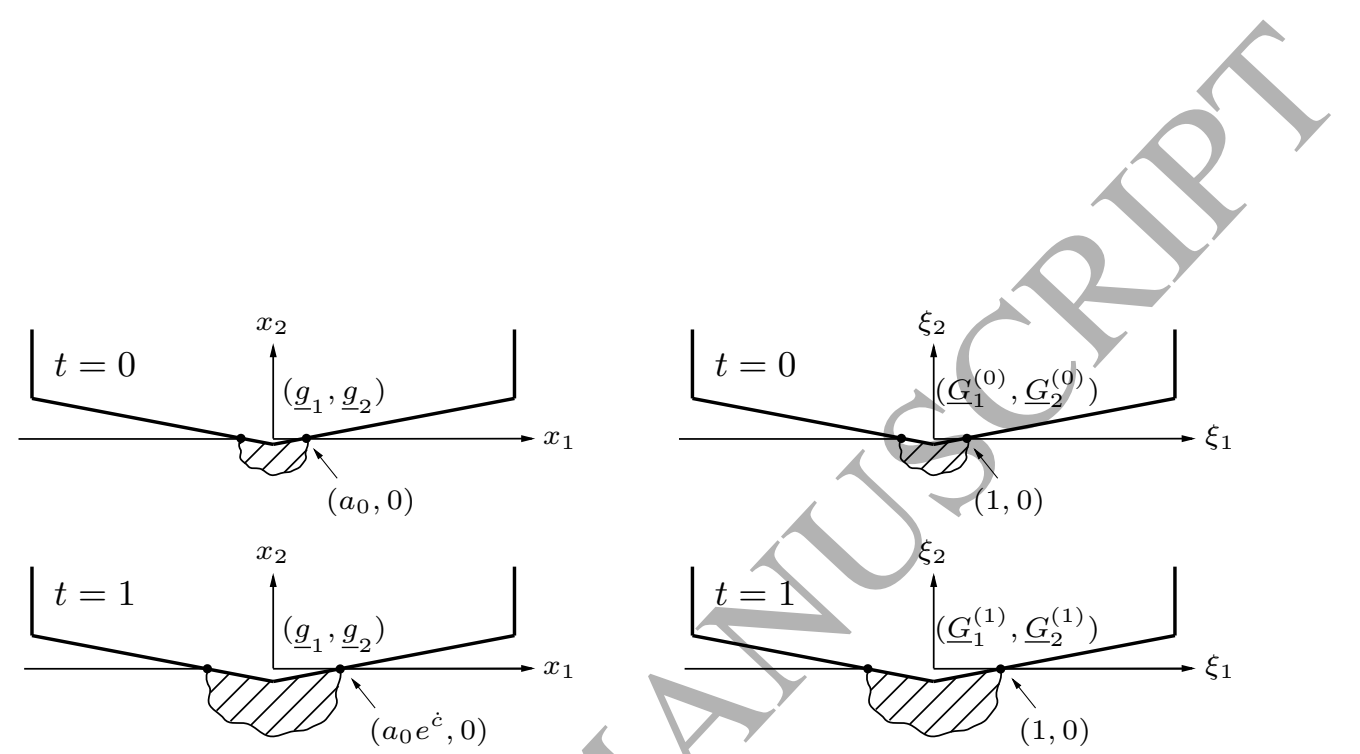

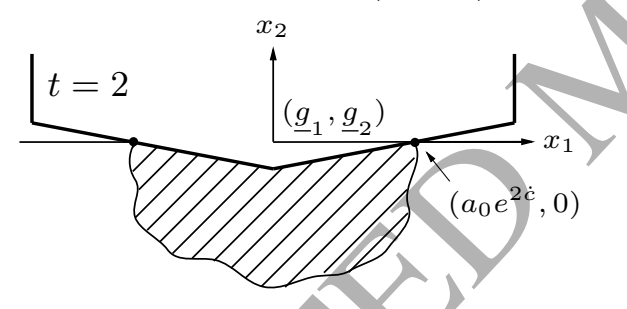

(a)
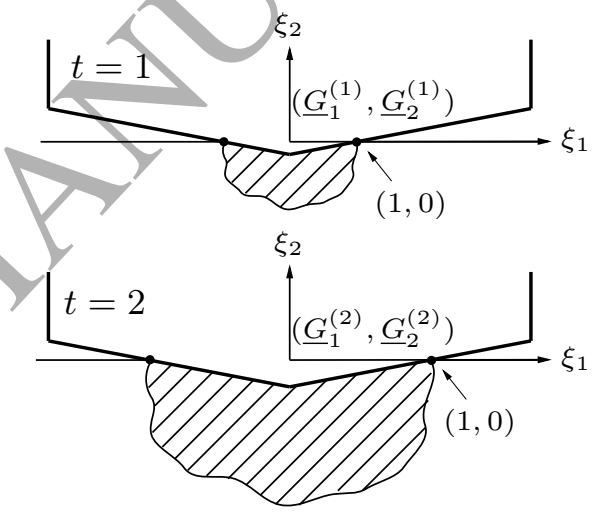

(b)

Figure 4: Development of self-similarity through time in (a) the reference coordinates with the basis vectors $\left(\underline{g}_{1}, \underline{g}_{2}\right)$, and in (b) the self-similar coordinate system with the stretched basis vectors $\left(\underline{G}_{1}^{(t)}, \underline{G}_{2}^{(t)}\right)$. 


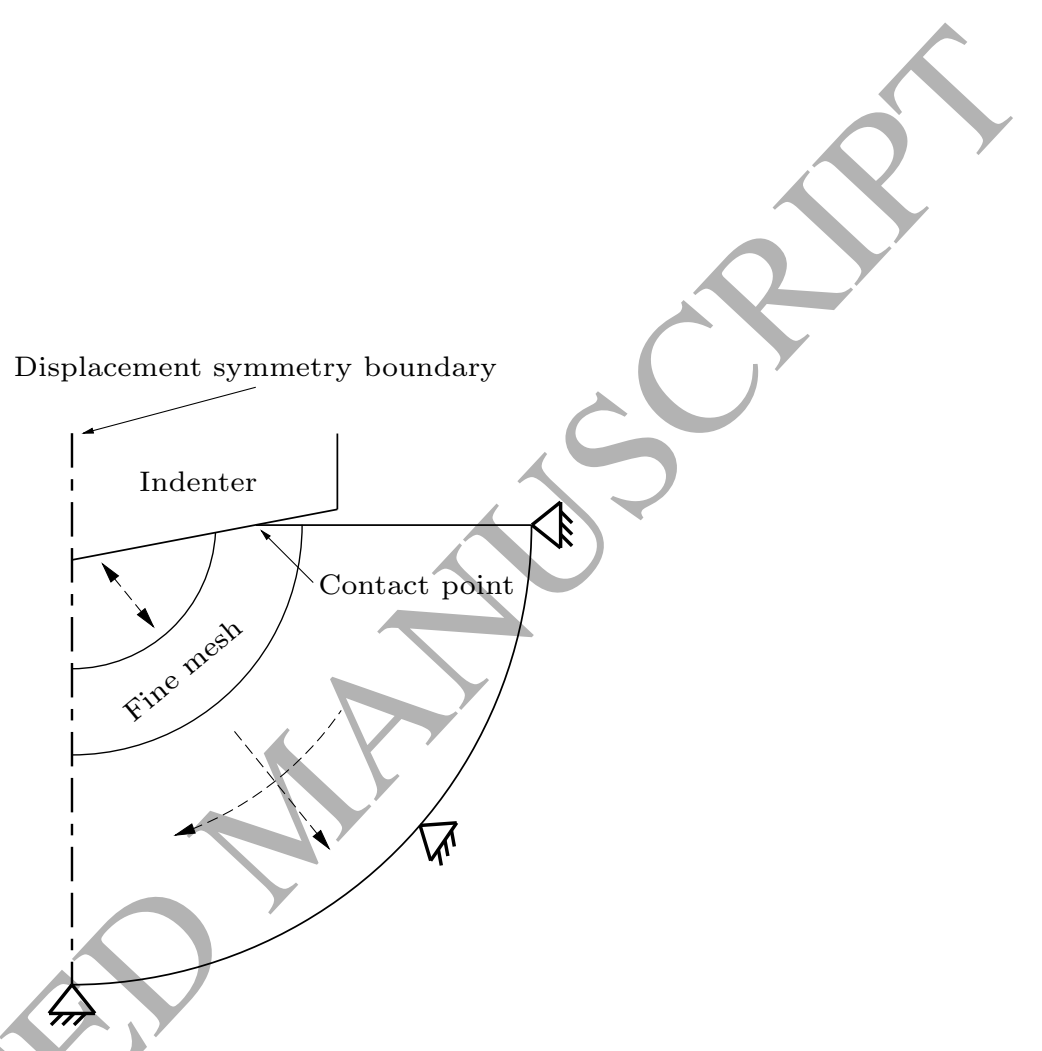

Figure 5: Right hand side of the domain used for numerical simulations. The dashed arrows indicate the direction of gradually increasing element size. The boundary of the domain is sufficiently far away from the contact point to have negligible influence on the results (the boundary is clamped).

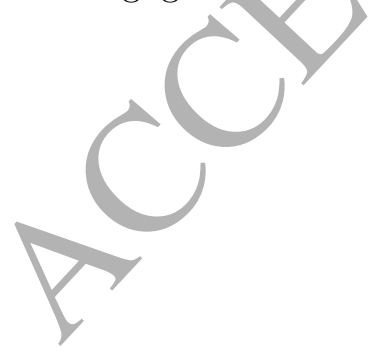




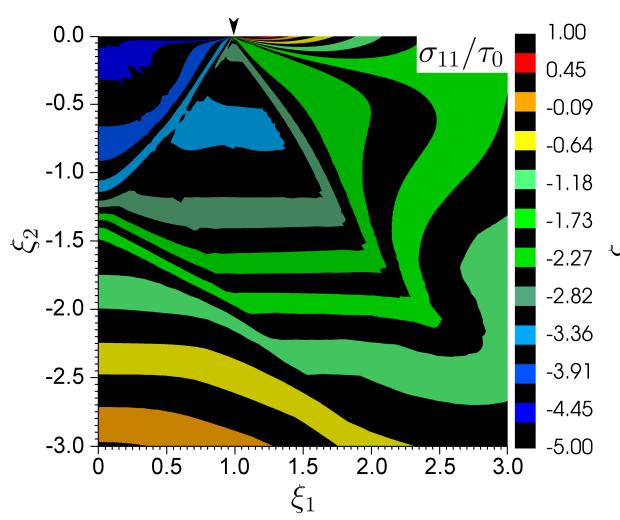

(a)

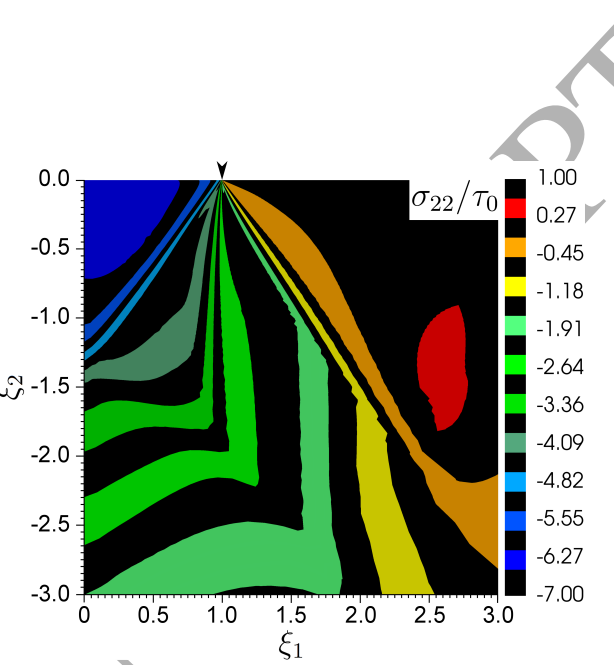

(b)

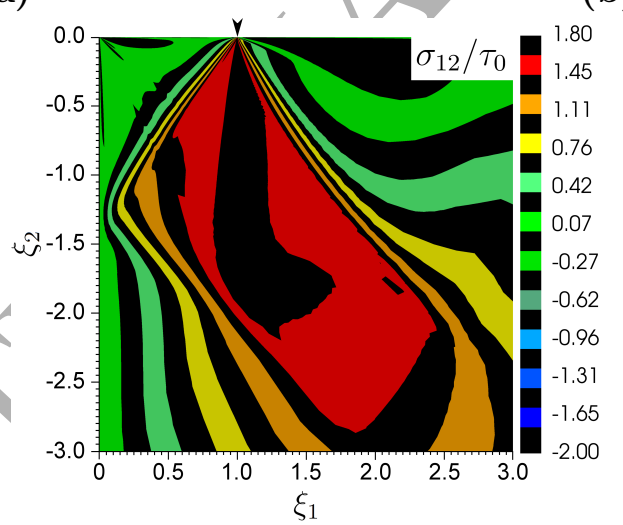

(c)

Figure 6: Stress distribution around the right hand side contact point, $\xi_{i}=(1,0)$, in FCC crystal for the stress components (a) $\sigma_{11} / \tau_{0}$, (b) $\sigma_{22} / \tau_{0}$ and (c) $\sigma_{12} / \tau_{0}$. 


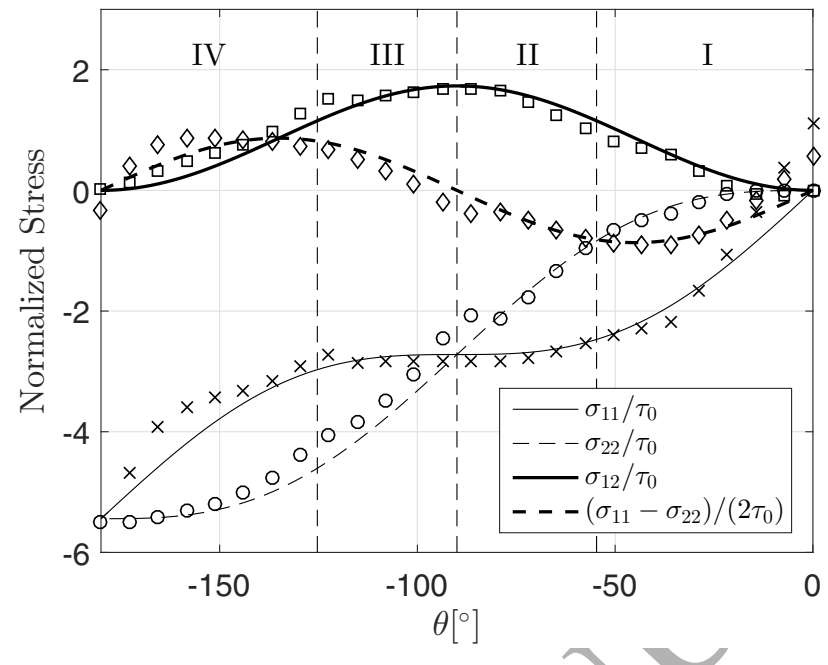

(a)

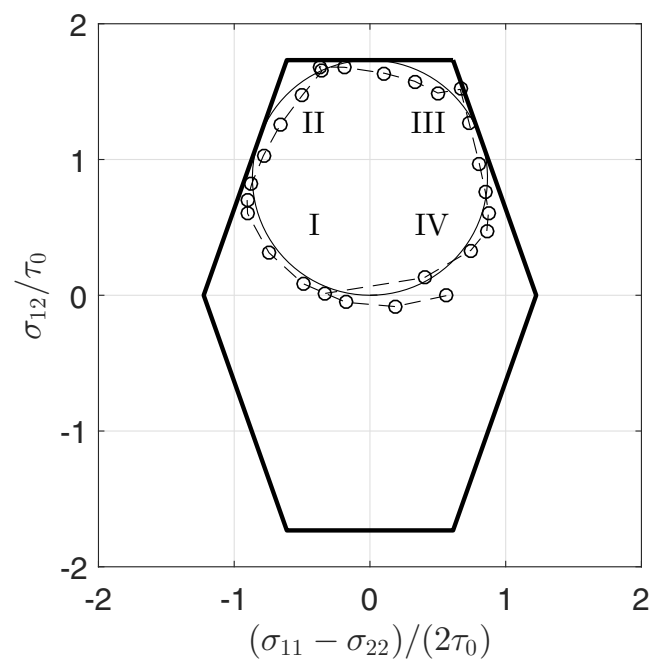

(b)

Figure 7: Stress distribution for FCC around the right hand side contact point projected as (a) angular distribution, and (b) stress trajectory with the thick line representing the yield surface. The lines represent the analytical solution while the markers indicate the numerical results. 


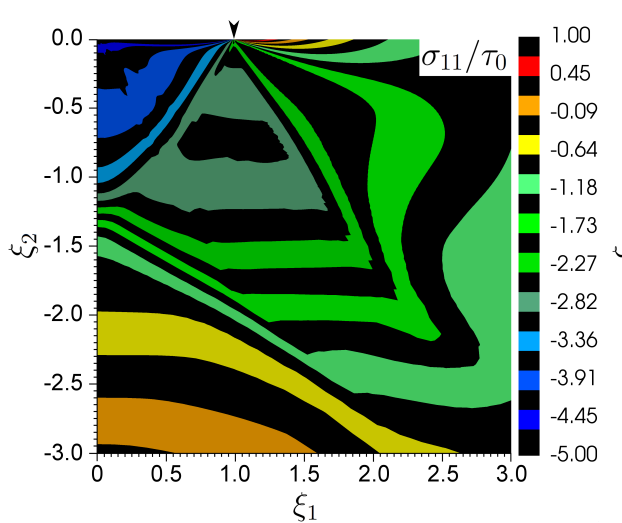

(a)

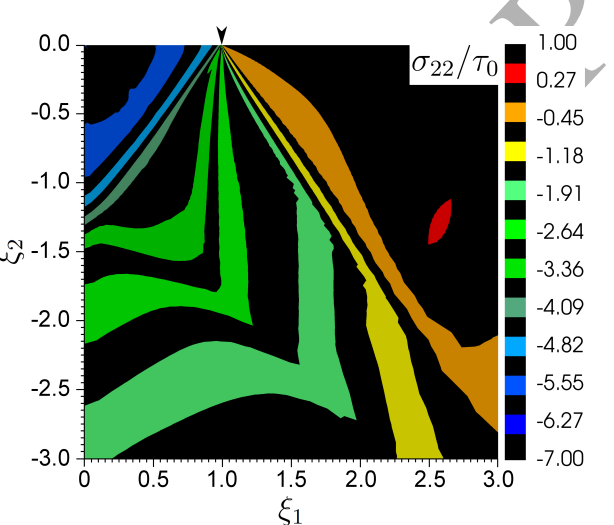

(b)

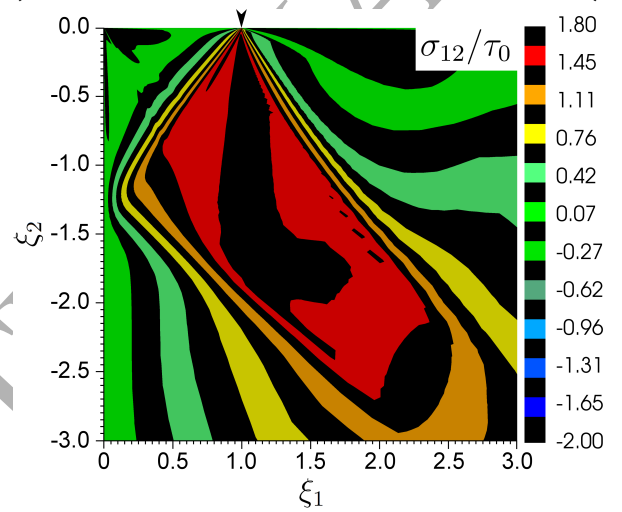

(c)

Figure 8: Stress distribution around the right hand side contact point, $\xi_{i}=(1,0)$, in BCC crystal for the stress components (a) $\sigma_{11} / \tau_{0}$, (b) $\sigma_{22} / \tau_{0}$ and (c) $\sigma_{12} / \tau_{0}$. 


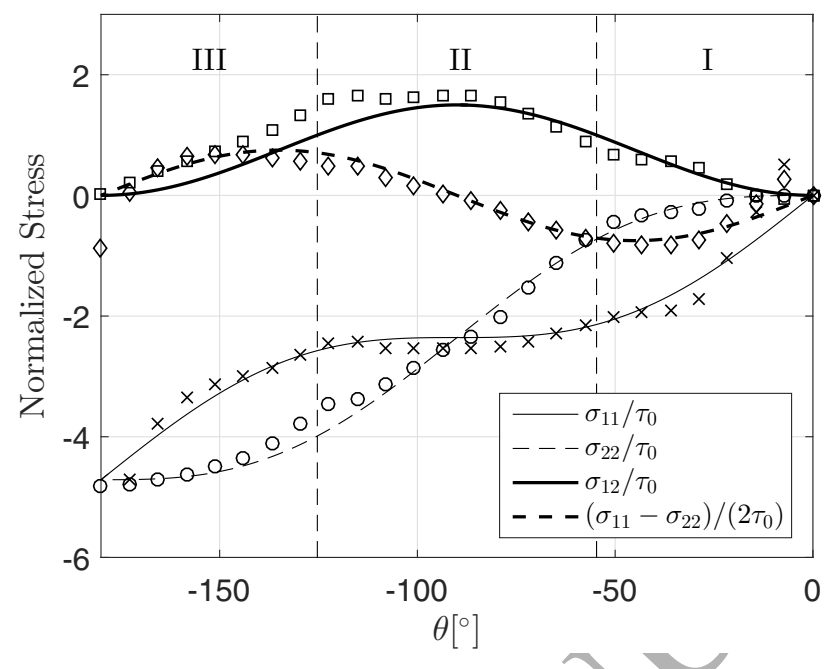

(a)

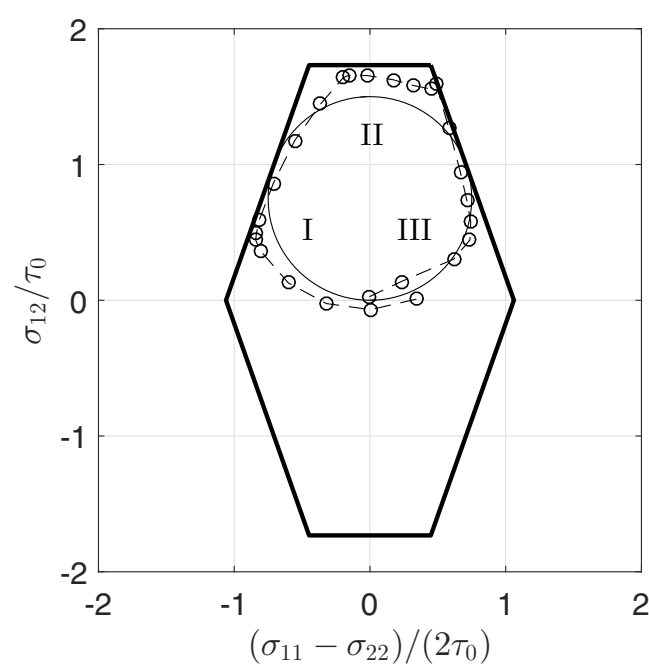

(b)

Figure 9: Stress distribution for $\mathrm{BCC}$ around the right hand side contact point projected as (a) angular distribution, and (b) stress trajectory with the thick line representing the yield surface. The lines represent the analytical solution while the markers indicate the numerical results. 


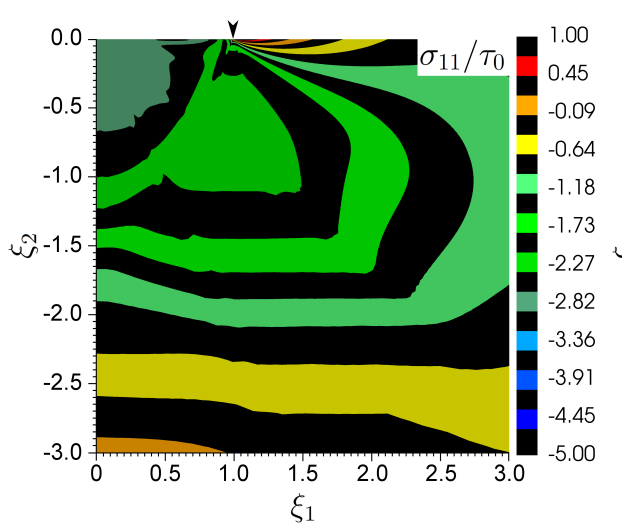

(a)

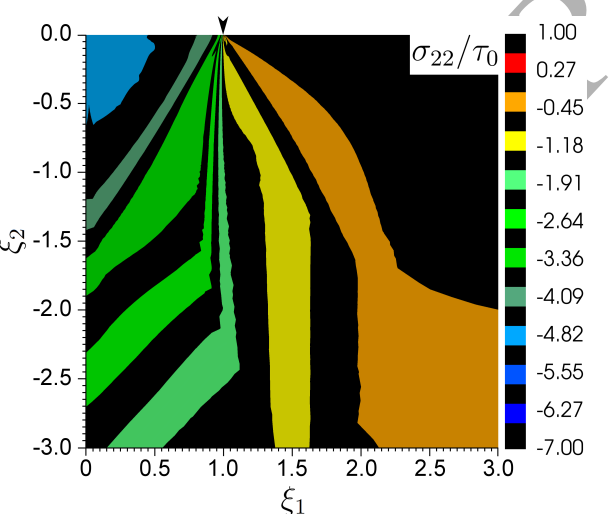

(b)

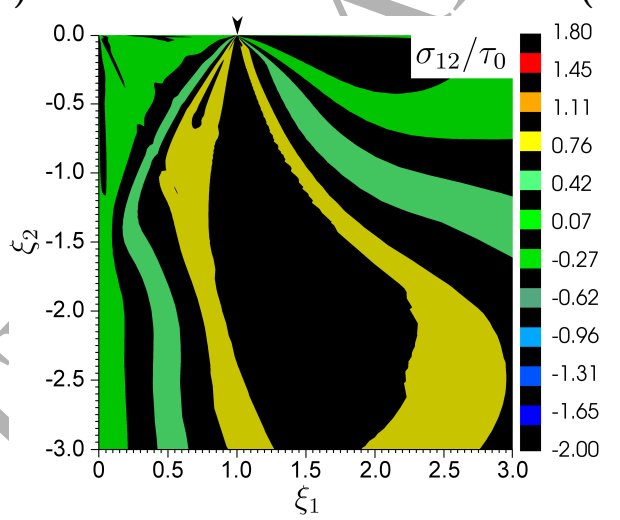

(c)

Figure 10: Stress distribution around the right hand side contact point, $\xi_{i}=$ $(1,0)$, in HCP crystal for the stress components (a) $\sigma_{11} / \tau_{0}$, (b) $\sigma_{22} / \tau_{0}$ and (c) $\sigma_{12} / \tau_{0} \cdot$ 

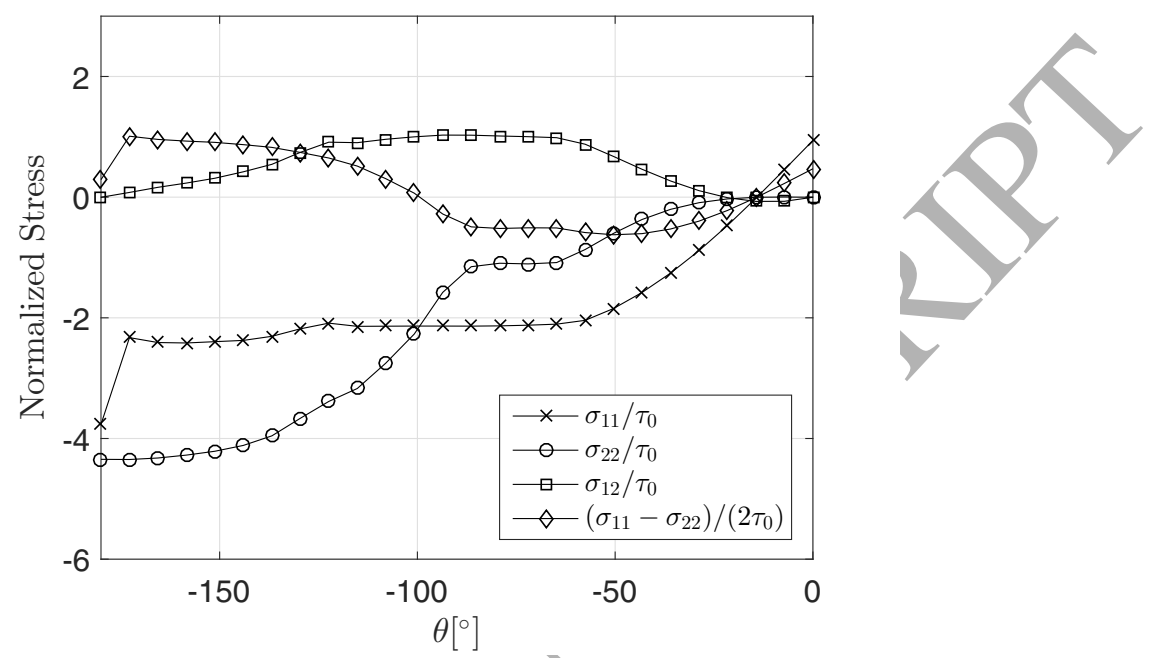

(a)

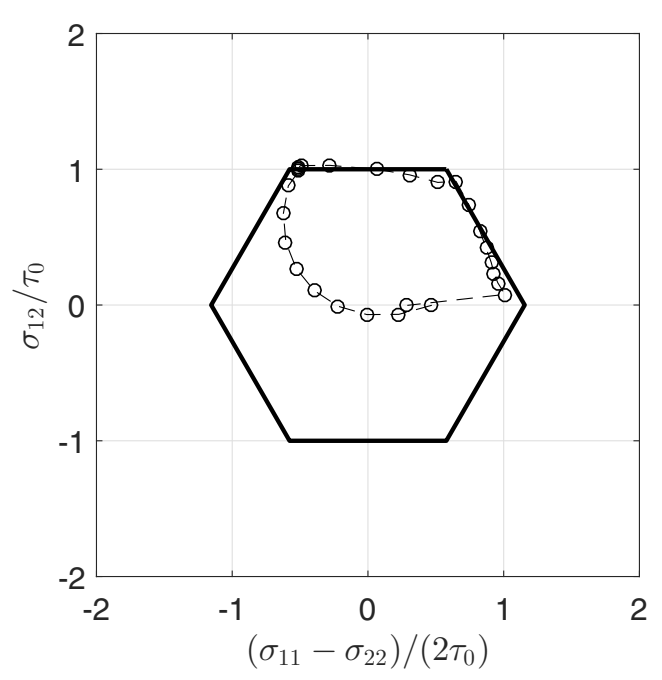

(b)

Figure 11: Stress distribution for HCP around the right hand side contact point projected as (a) angular distribution, and (b) stress trajectory with the thick line representing the yield surface. Here, only the numerical solution is presented. 


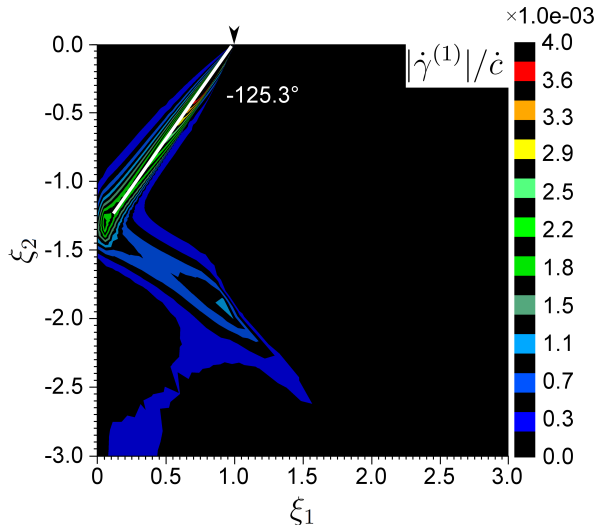

(a)

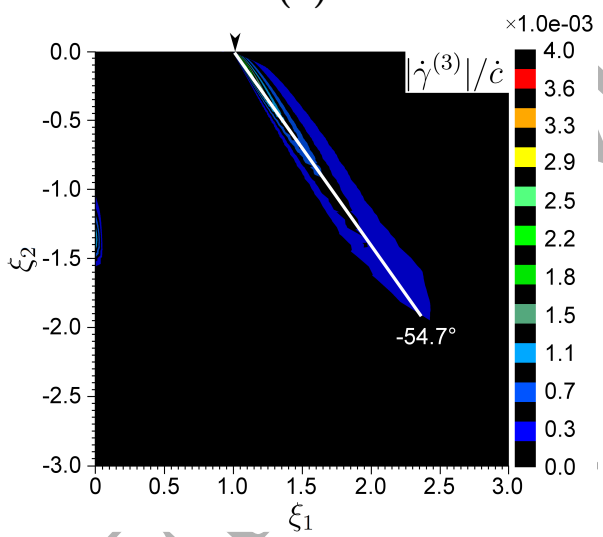

(c)

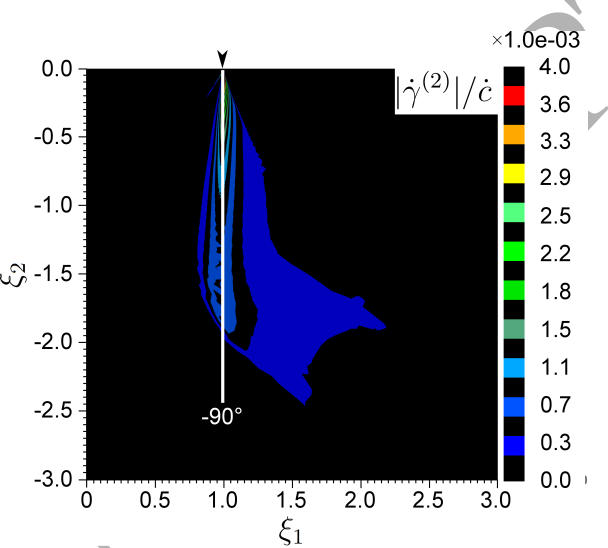

(b)

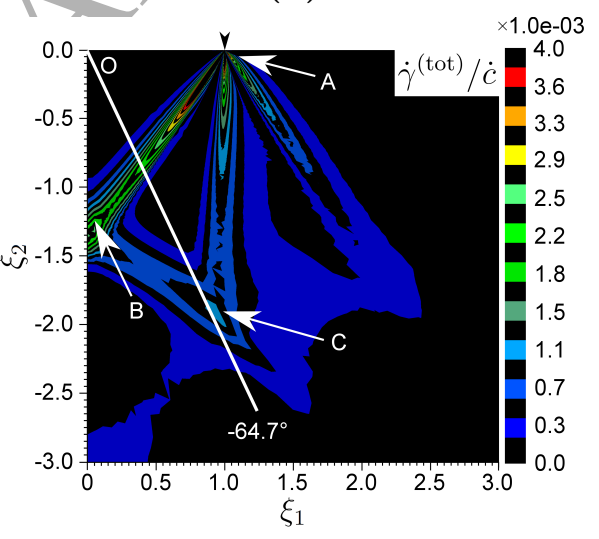

(d)

Figure 12: Slip rate around the right hand side contact point, $\xi_{i}=(1,0)$, in FCC crystal for the slip systems (a) $\left|\dot{\gamma}^{(1)}\right| / \dot{c}$, (b) $\left|\dot{\gamma}^{(2)}\right| / \dot{c}$, (c) $\left|\dot{\gamma}^{(3)}\right| / \dot{c}$, and (d) $\dot{\gamma}^{\text {(tot) } / \dot{c}}$ $\left(\dot{\gamma}^{\text {(tot })}=\sum^{\alpha}\left|\dot{\gamma}^{(\alpha)}\right|\right)$. 


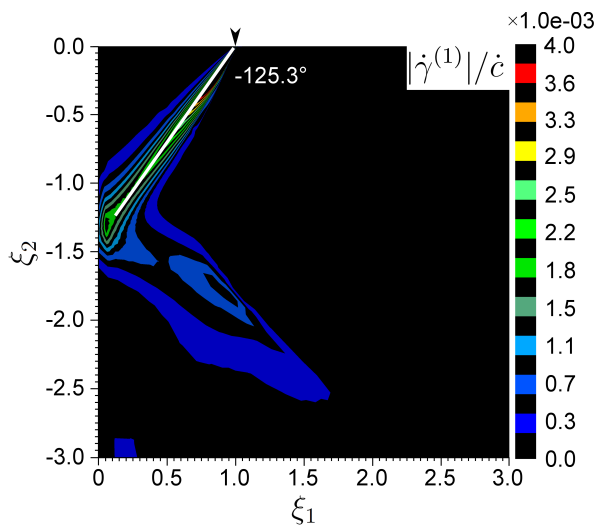

(a)

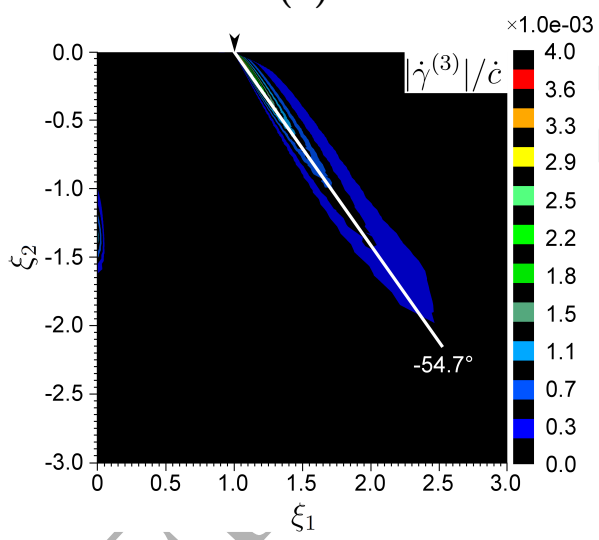

(c)

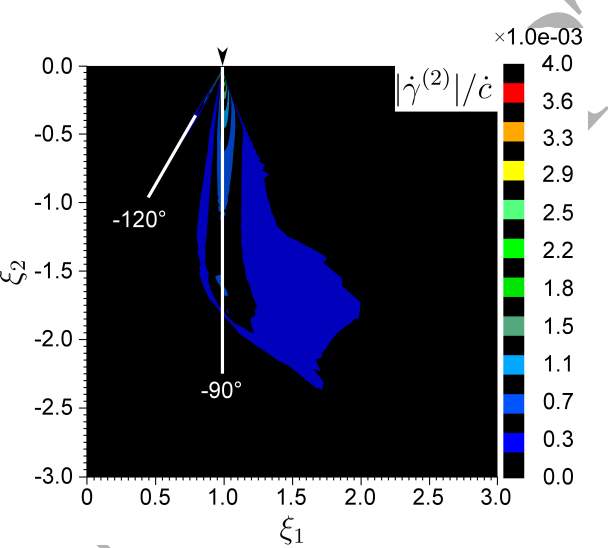

(b)

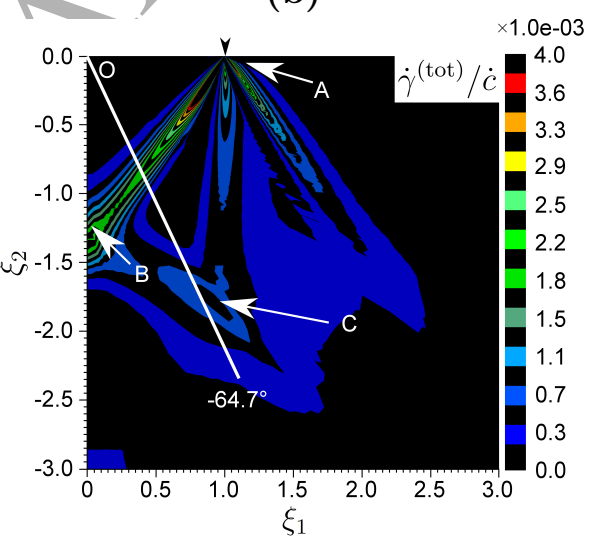

(d)

Figure 13:) Slip rate around the right hand side contact point, $\xi_{i}=(1,0)$, in BCC crystal for the slip systems (a) $\left|\dot{\gamma}^{(1)}\right| / \dot{c}$, (b) $\left|\dot{\gamma}^{(2)}\right| / \dot{c}$, (c) $\left|\dot{\gamma}^{(3)}\right| / \dot{c}$, and (d) $\dot{\gamma}^{(\text {tot })} / \dot{c}$ $\left(\dot{\gamma}^{\text {(tot })} \neq \sum^{\alpha}\left|\dot{\gamma}^{(\alpha)}\right|\right)$. 


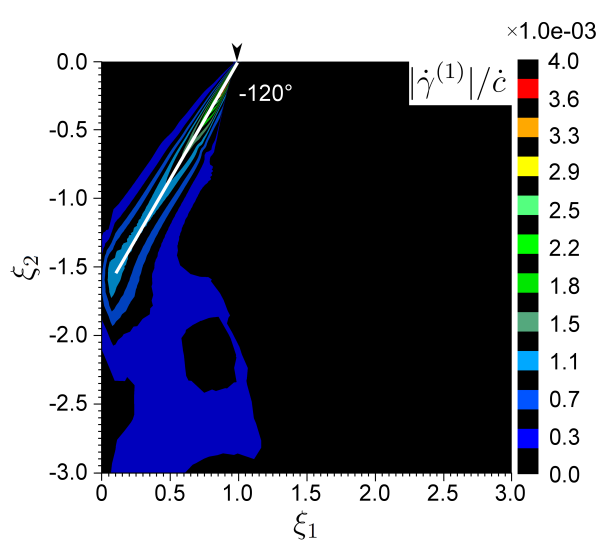

(a)

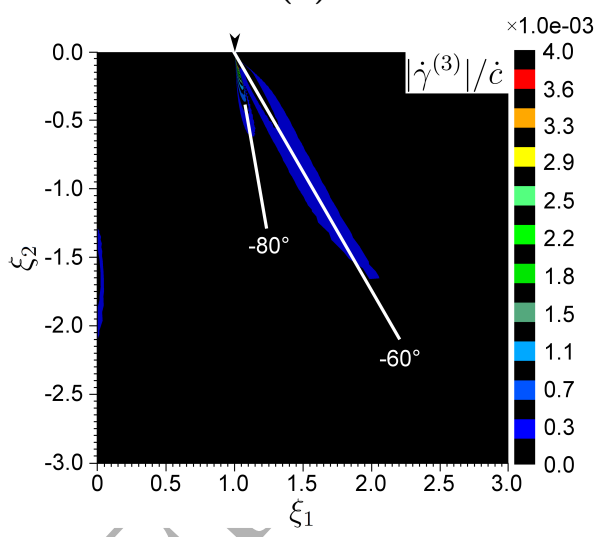

(c)

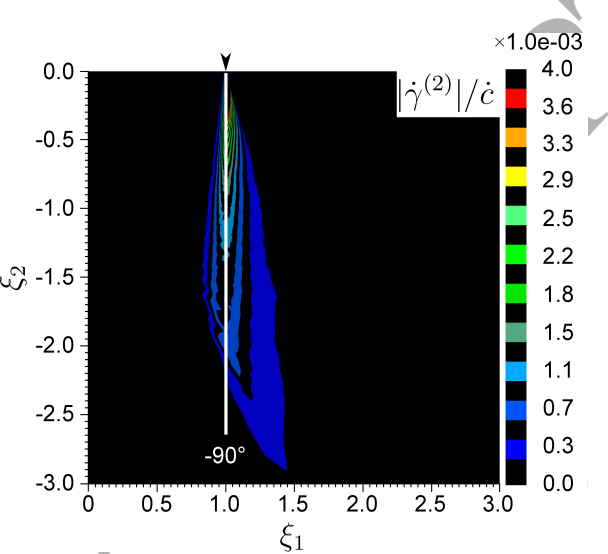

(b)

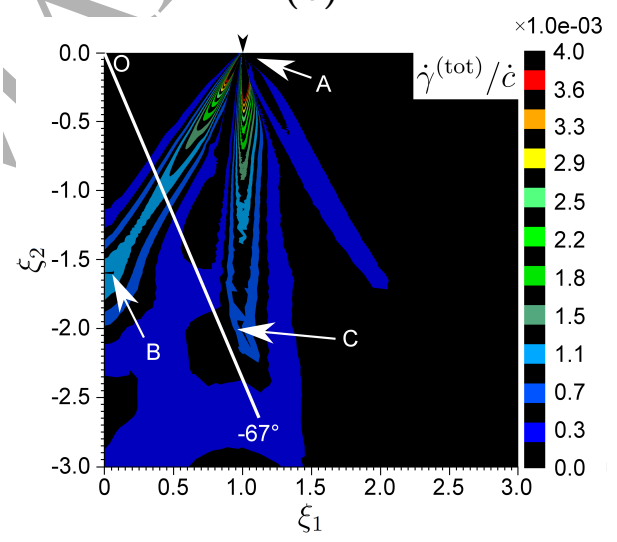

(d)

Figure 14: Slip rate around the right hand side contact point, $\xi_{i}=(1,0)$, in HCP crystal for the slip systems (a) $\left|\dot{\gamma}^{(1)}\right| / \dot{c}$, (b) $\left|\dot{\gamma}^{(2)}\right| / \dot{c}$, (c) $\left|\dot{\gamma}^{(3)}\right| / \dot{c}$, and (d) $\dot{\gamma}^{\text {(tot) }} / \dot{c}$ $\left(\dot{\gamma}^{\text {(tot })} \neq \sum^{\alpha}\left|\dot{\gamma}^{(\alpha)}\right|\right)$. 


\section{${ }_{735}$ List of Tables}

${ }_{736} \quad 1 \quad$ Model parameters. . . . . . . . . . . . . 52

${ }_{737} 2$ Effective slip systems for plane strain model. . . . . . . . . 53

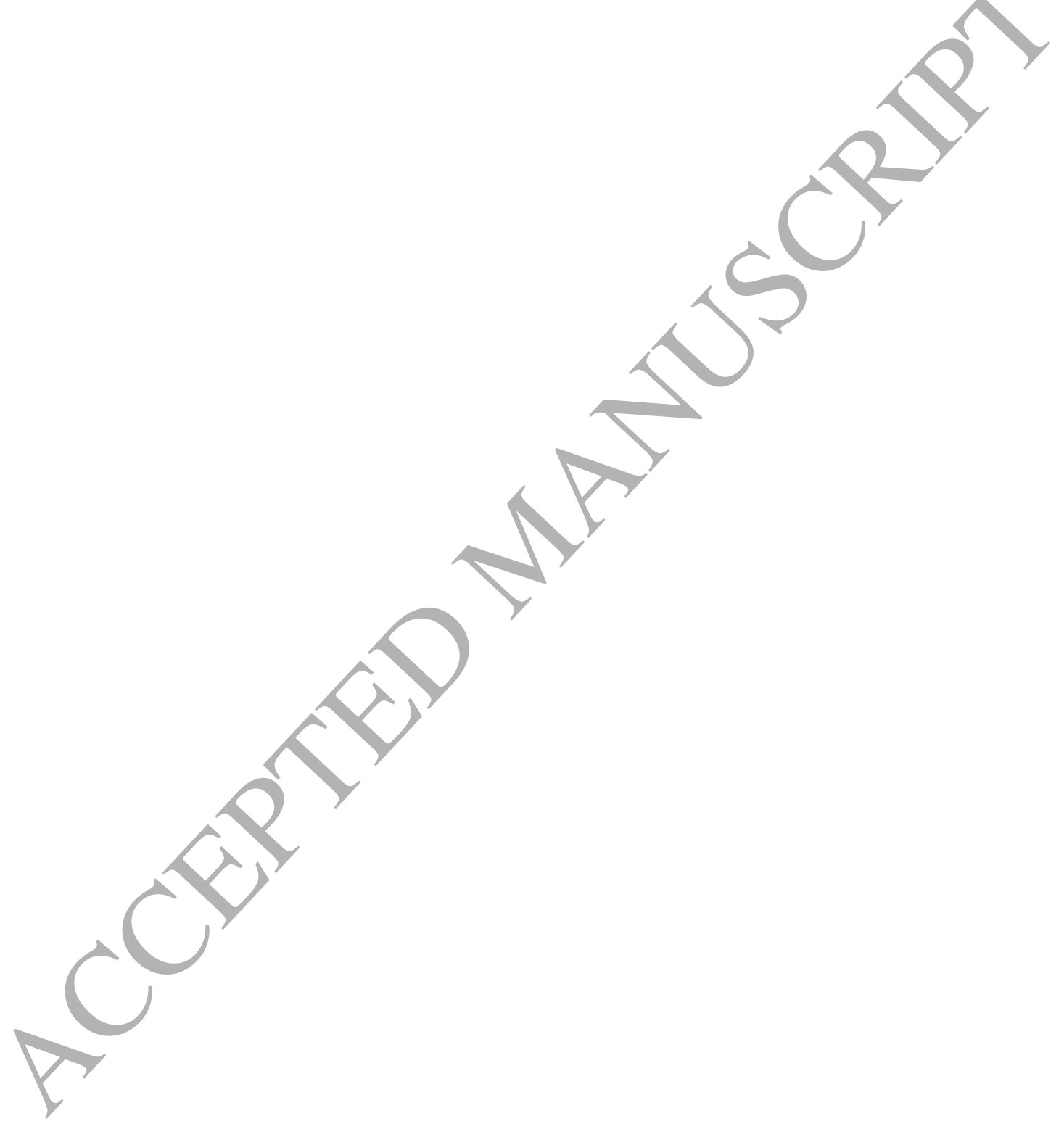




\begin{tabular}{cll}
\hline Parameter & Significance & Value \\
\hline$\tau_{0} / E$ & Yield strain & $\sim 1 \mathrm{e}^{-5}$ \\
$\nu$ & Poisson ratio & 0.3 \\
$m$ & Strain rate-sensitivity exponent & 0.02 \\
$\dot{\gamma}_{0}$ & Reference slip rate & $0.001 \mathrm{~s}^{-1}$ \\
$\dot{c}$ & Magnification rate & $0.1 \mathrm{~s}^{-1}$ \\
$\phi$ & Indenter angle & $0.038^{\circ}$ \\
\hline
\end{tabular}

Table 1: Model parameters.

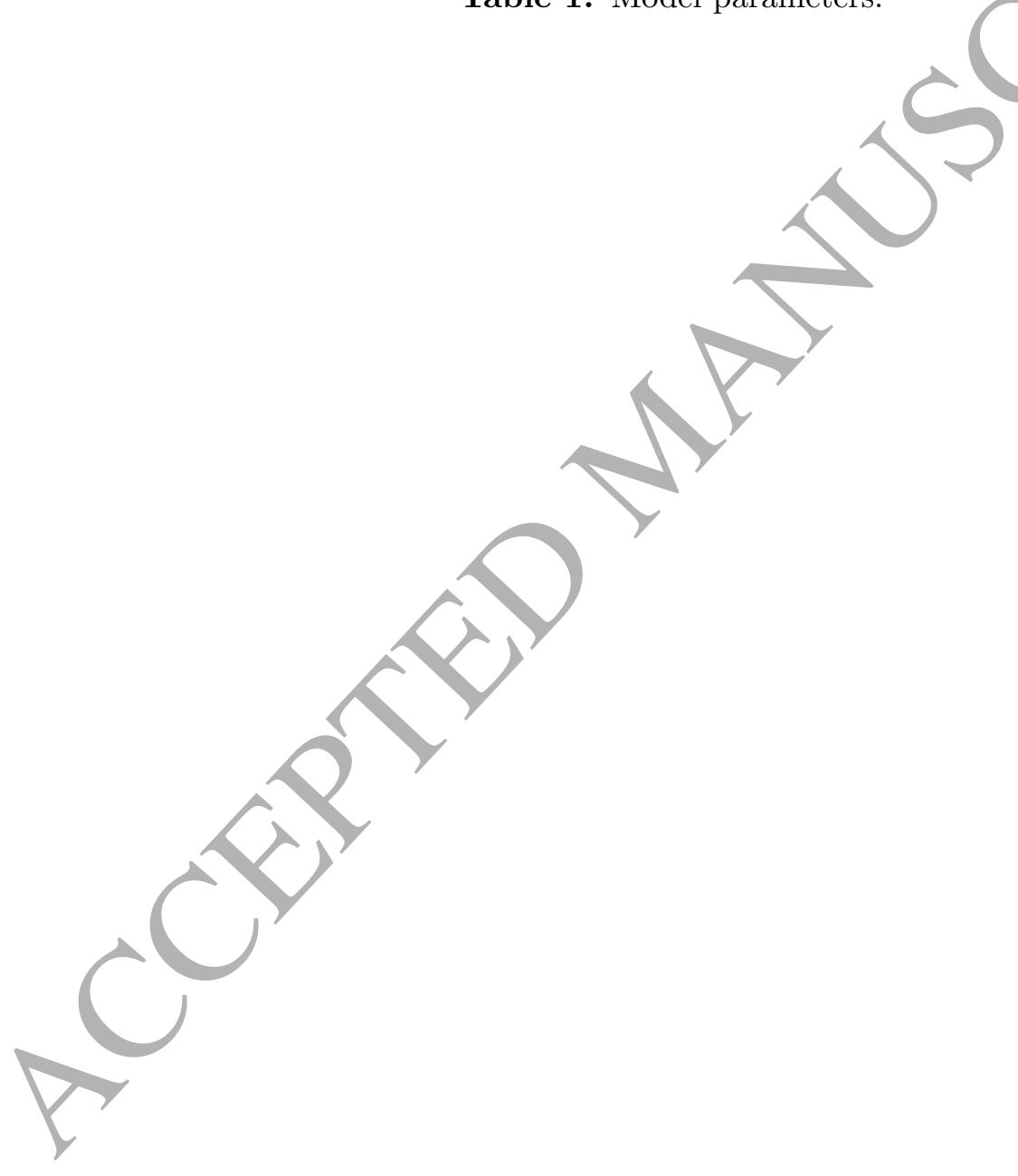




\begin{tabular}{|c|c|c|c|}
\hline Effective slip system no. & (1) & $(2)$ & (3) \\
\hline \multicolumn{4}{|c|}{ FCC } \\
\hline Angle to [101] in ( $\overline{101})$ plane & $54.7^{\circ}$ & $0^{\circ}$ & $-54.7^{\circ}$ \\
\hline Crystallographic slip system (a) & $(111)[1 \overline{1} 0]$ & $(11 \overline{1})[101]$ & 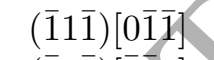 \\
\hline Crystallographic slip system (b) & $(111)[0 \overline{1} 1]$ & $(\overline{1} 11)[101]$ & \\
\hline$\beta^{(\alpha)}=\frac{s_{i}^{(\alpha a)} m_{j}^{(\alpha a)}+s_{i}^{(\alpha b)} m_{j}^{(\alpha b)}}{s_{i}^{(\alpha)} m_{i}^{(\alpha)}}$ & \multicolumn{3}{|l|}{$\sqrt{3}$} \\
\hline$\lambda^{(\alpha)}=\frac{\tau^{(\alpha)}}{\tau^{(\alpha a)}}=\frac{\tau^{(\alpha)}}{\tau^{(\alpha b)}}$ & \multicolumn{3}{|l|}{$\frac{2}{\sqrt{3}}$} \\
\hline \multicolumn{4}{|c|}{$\mathrm{BCC}$} \\
\hline Angle to $[010]$ in $(\overline{101})$ plane $\left[^{\circ}\right]$ & 54.7 & & $-54.7^{\circ}$ \\
\hline Crystallographic slip system (a) & $(\overline{1} \overline{2} \overline{1})[1 \overline{1} 1]$ & $(101)[\overline{1} \overline{1} 1]$ & $(\overline{1} 2 \overline{1})[111]$ \\
\hline Crystallographic slip system (b) & & 01) [111] & - \\
\hline$\beta^{(\alpha)}=\frac{s_{i}^{(\alpha a)} m_{j}^{(\alpha a)}+s_{i}^{(\alpha b)} m_{j}^{(\alpha b)}}{s_{i}^{(\alpha)} m_{j}^{(\alpha)}}$ & & $\frac{2}{\sqrt{3}}$ & 1 \\
\hline$\lambda^{(\alpha)}=\frac{\tau^{(\alpha)}}{\tau^{(\alpha a)}}=\frac{\tau^{(\alpha)}}{\tau^{(\alpha b)}}$ & & $\sqrt{3}$ & 1 \\
\hline Angle to $[11 \overline{2} 0]$ in $(0001)$ plane & $60^{\circ}$ & $0^{\circ}$ & $-60^{\circ}$ \\
\hline Crystallographic slip system (a) & $(10 \overline{1} 0)[1 \overline{2} 10]$ & $(1 \overline{1} 00)[\overline{1} \overline{1} 20]$ & $(01 \overline{1} 0)[2 \overline{1} \overline{1} 0]$ \\
\hline Crystallographic slip system (b) & - & - & - \\
\hline$\beta^{(\alpha)}$ & 1 & 1 & 1 \\
\hline$\lambda^{(\alpha)}=\frac{\tau^{(\alpha)}}{\tau^{(\alpha a)}}=\frac{\tau^{(\alpha)}}{\tau^{(\alpha b)}}$ & 1 & 1 & 1 \\
\hline
\end{tabular}

Table 2: Effective slip systems for plane strain model. 